\title{
Quasifinite Highest Weight Modules over the Lie Algebra of Differential Operators on the Circle
}

\author{
Victor Kac ${ }^{\star}$, Andrey Radul ${ }^{\star \star}$ \\ Mathematics Department, M.I.T., Cambridge, MA 02139, USA
}

Received: 7 June 1993

\begin{abstract}
We classify positive energy representations with finite degeneracies of the Lie algebra $W_{1+\infty}$ and construct them in terms of representation theory of the Lie algebra $\hat{g} l\left(\infty, R_{m}\right)$ of infinites matrices with finite number of non-zero diagonals over the algebra $R_{m}=\mathbb{C}[t] /\left(t^{m+1}\right)$. The unitary ones are classified as well. Similar results are obtained for the sin-algebras.
\end{abstract}

\section{Introduction}

0.1. Recent progress in conformal field theory revealed some unusual mathematical objects called the $W_{n}$-algebras [Z]. These algebras turned out to be quantizations of the second Gelfand-Dickey structure for Lax equations [FL]. The complicated structure of these algebras is greatly simplified in the limit $n=\infty$, the limiting algebra being the Lie algebra $\mathscr{\mathscr { D }}$, the universal central extension of the Lie algebra of differential operators on the circle [KP]. (Physicists denote this Lie algebra by $W_{1+\infty}$ [PSR].) The possibility to get $W_{n}$ from $\widehat{\mathscr{D}}$ has been studied in [R, RV]. A complete picture for classical $W_{n}$ was obtained in [KhZ].

The main goal of the present paper is to classify and describe the irreducible quasifinite highest weight representation of the Lie algebra $\widehat{\mathscr{D}}$. The basic technical tool is the analytic completion $\widehat{\mathscr{D} \mathscr{C}}$ of $\widehat{\mathscr{D}}$ and a family of its homomorphisms onto the central extension of the Lie algebra $\widetilde{g l}\left(\infty, R_{m}\right)$ of infinite matrices with finitely many non-zero diagonals over the ring $R_{m}=\mathbb{C}[t] /\left(t^{m+1}\right)$.

The Lie algebra $\widehat{\mathscr{D}}$ may be obtained via a general construction (explained in Sect. 1) as a twisted Laurent polynomial algebra over the polynomial algebra $\mathbb{C}[w]$. It is easy to see that the only other Lie algebras obtained by this construction from $\mathbb{C}[w]$ are Lie algebras $\widehat{\mathscr{D}}_{q}$, the central extension of the Lie algebra of difference

* Supported in part by NSF grant DMS-9103792

$\star \star$ Supported in part by DOE grant DE-F602-88ER25066 
operators on the circle. It turns out, however, that a representation theory similar to that of $\widehat{\mathscr{D}}$, may be developed for a larger Lie algebra, the central extension $\widehat{\mathscr{S}}$ of the Lie algebra of pseudo-difference operators on the circle (see Sect. 6). The latter Lie algebra has been studied recently by many authors (see [FFZ, GL] and references there).

Being of a very general nature, our methods may be applied to many other examples of infinite-dimensional Lie algebras. Noting that $\mathscr{D}\left(\right.$ resp. $\mathscr{S}_{q}$ ) is a quantization of the Poisson Lie algebra of functions on the cylinder (resp. 2-dimensional tours), one may expect that our approach could be extended to the quantizations of general symplectic manifolds.

0.2 . Let us give here the main definitions which will be used for various examples throughout the paper.

Consider a $\mathbb{Z}$-graded Lie algebra over $\mathbb{C}$ :

$$
\mathfrak{g}=\bigoplus_{j \in \mathbb{Z}} \mathfrak{g}_{j}, \quad\left[\mathfrak{g}_{i}, \mathfrak{g}_{j}\right] \subset \mathfrak{g}_{i+j}
$$

(We do not assume $\mathfrak{g}_{2}$ to be finite-dimensional.) We let

$$
\mathfrak{g}_{ \pm}=\bigoplus_{j>0} \mathfrak{g}_{ \pm j}
$$

A subalgebra $\mathfrak{p}$ of $\mathfrak{g}$ is called parabolic if it contains $\mathfrak{g}_{0}+\mathfrak{g}_{+}$as a proper subalgebra.

A $\mathfrak{g}$-module $V$ is called graded if

$$
V=\bigoplus_{\jmath} V_{j}, \quad \mathfrak{g}_{i} V_{j} \subset V_{i+j} .
$$

A graded $\mathfrak{g}$-module $V$ is called quasifinite if

$$
\operatorname{dim} V_{j}<\infty \text { for all } j .
$$

Given $\lambda \in \mathfrak{g}_{0}^{*}$, a highest weight module is a $\mathbb{Z}$-graded $\mathfrak{g}$-module $V(\mathfrak{g}, \lambda)=$ $\bigoplus_{j \in \mathbb{Z}_{+}} V_{-j}$ defined by the following properties:

(i) $V_{0}=\mathbb{C} v_{\lambda}$, where $v_{\lambda}$ is a non-zero vector,

(ii) $h v_{\lambda}=\lambda(h) v_{\lambda}$ for $h \in \mathfrak{g}_{0}$,

(iii) $\mathfrak{g}_{+} v_{\lambda}=0$,

(iv) $\mathscr{\mathscr { C }}\left(\mathfrak{g}_{-}\right) v_{\lambda}=V(\mathfrak{g}, \lambda)$.

Here and further $\mathscr{W}(\mathfrak{s})$ stands for the universal enveloping algebra of the Lie algebra $\mathfrak{s}$.

A non-zero vector $v \in V(\mathfrak{g}, \lambda)$ is called singular if $\mathfrak{g}_{+} v=0$. The module $V(\mathfrak{g}, \lambda)$ is irreducible if and only if any of its singular vectors is a multiple of $v_{\lambda}$.

The "largest" among the modules $V(\mathfrak{g}, \lambda)$ with a given $\lambda$ is the Verma module $M(\mathfrak{g}, \lambda)$ defined by the property that the map

$$
\varphi: \mathscr{C}\left(\mathfrak{g}_{-}\right) \rightarrow M(\mathfrak{g}, \lambda)
$$

given by $\varphi(u)=u\left(v_{\lambda}\right)$ is a vector space isomorphism.

Any highest weight module $V(\mathfrak{g}, \lambda)$ is a quotient of $M(\mathfrak{g}, \lambda)$. The "smallest" among the $V(\mathfrak{g}, \lambda)$ is the irreducible module $L(\mathfrak{g}, \lambda)$ (which is a quotient of $M(\mathfrak{g}, \lambda)$ by the maximal graded submodule). 
We shall write $M(\lambda)$ and $L(\lambda)$ in place of $M(\mathfrak{g}, \lambda)$ and $L(\mathfrak{g}, \lambda)$ if no ambiguity may arise.

0.3. It is useful to note that the Verma modules can be constructed as follows:

$$
M(\mathfrak{g}, \lambda)=\mathscr{Z}(\mathfrak{g}) \otimes_{\mathscr{C}\left(\mathfrak{g}_{0}+\mathfrak{g}_{+}\right)} \mathbb{C}_{\lambda},
$$

where $\mathbb{C}_{\lambda}$ is the 1-dimensional $\mathfrak{g}_{0}+\mathfrak{g}_{+}$-module given by $h \mapsto \lambda(h)$ if $h \in \mathfrak{g}_{0}, \mathfrak{g}_{+} \mapsto 0$, and the action of $\mathfrak{g}$ is induced by the left multiplication in $\mathscr{C}(\mathfrak{g})$.

Now, let $\mathfrak{p}=\oplus_{j} \mathfrak{p}_{j}$ be a parabolic subalgebra of $\mathfrak{g}$, and let $\lambda \in \mathfrak{g}_{0}^{*}$ be such that $\left.\lambda\right|_{\mathfrak{g}_{0} \cap[p, p]}=0$. Then the $\mathfrak{g}_{0}+\mathfrak{g}_{+}$-module $\mathbb{C}_{\lambda}$ extends to $\mathfrak{p}$ by letting $\mathfrak{p}_{j} \mapsto 0$ for $j<0$, and we may construct the highest weight module

$$
M(\mathfrak{g}, \mathfrak{p}, \lambda)=\mathscr{Q} b(\mathfrak{g}) \otimes_{\mathscr{Z}(\mathfrak{p})} \mathbb{C}_{\lambda}
$$

It is called the generalized Verma module. It may be characterized by the property that the map $\varphi$ induces an isomorphism $\mathscr{\mathscr { C }}\left(\mathfrak{g}_{-}\right) / \mathscr{U}\left(\mathfrak{p} \cap \mathfrak{g}_{-}\right) \rightarrow M(\mathfrak{g}, \mathfrak{p}, \lambda)$.

Note that if $\operatorname{dim} \mathfrak{g}_{\jmath}<\infty$ for all $j$, the $\mathfrak{g}$-module $L(\lambda)$ for any $\lambda$ is quasifinite. If however $\operatorname{dim} \mathfrak{g}_{j}=\infty$, which is the case in all of our examples, the classification of quasifinite irreducible highest weight modules becomes a non-trivial problem. The answer to this problem for the Lie algebra $\widehat{\mathscr{D}}$ is given by Theorem 4.2. Moreover, we give an explicit construction of all these modules in terms of irreducible highest weight modules over the Lie algebra $\widehat{g l}\left(\infty, R_{m}\right)$ (Theorems 4.5 and 4.6).

0.4. Recall that an anti-involution of a Lie algebra $\mathfrak{g}$ over $\mathbb{C}$ is an additive map $\omega: \mathfrak{g} \rightarrow \mathfrak{g}$ such that

$$
\omega(\lambda a)=\bar{\lambda} a, \quad \omega([a, b])=[\omega(b), \omega(a)], \quad \text { for } \lambda \in \mathbb{C}, \quad a, b \in \mathfrak{g} .
$$

Given a $\mathfrak{g}$-module $V$, a Hermitian form $h$ on $V$ is called contravariant if for any $a \in \mathfrak{g}$ the operators $a$ and $\omega(a)$ are (formally) adjoint operators on $V$ with respect to $h$.

Fix an anti-involution $\omega$ of the Lie algebra $\mathfrak{g}$ such that $\omega\left(\mathfrak{g}_{j}\right)=\mathfrak{g}_{-j}$. Let $L(\mathfrak{g}, \lambda)$ be an irreducible highest weight module over $\mathfrak{g}$ such that $\lambda(h) \in \mathbb{R}$ if $\omega(h)=h$. For $v \in L(\mathfrak{g}, \lambda)$ denote by $\langle v\rangle$ the coefficient of $v_{\lambda}$ in the decomposition of $v$ with respect to the gradation of $L(\mathfrak{g}, \lambda)$. Let

$$
h\left(a v_{\lambda}, b v_{\lambda}\right)=\left\langle\omega(a) b v_{\lambda}\right\rangle, \quad a, b \in \mathscr{\mathscr { b }}(\mathfrak{g}) .
$$

It is easy to show (see e.g. [K, Chapter 9]) that $h$ is the unique contravariant form on $L(\mathfrak{g}, \lambda)$ such that $h\left(v_{\lambda}, v_{\lambda}\right)=1$; moreover, it is non-degenerate and $h\left(L(\mathfrak{g}, \lambda)_{\imath}\right.$, $\left.L(\mathfrak{g}, \lambda)_{\jmath}\right)=0$ if $i \neq j$.

The $\mathfrak{g}$-module $L(\mathfrak{g}, \lambda)$ is called unitary (with respect to $\omega$ ) if the contravariant form $h$ is positive definite (this is independent of the choice of $v_{\lambda} \in L(\mathfrak{g}, \lambda)_{0}$ ).

The classification of unitary quasifinite (irreducible) highest weight modules over $\widehat{\mathscr{D}}$ is given by Theorem 5.2 .

Let us note in conclusion that the classification of irreducible quasifinite highest weight $\widehat{\mathscr{D}}$-modules is expressed in terms of Bernoulli polynomials. Is it an indication of a connection to the Riemann-Roch theorems?

0.5. One of the authors wishes to thank D. Lebedev and M. Golenishcheva-Kutuzova for illuminating discussions on the sin Lie algebra. 


\section{Twisted Laurent Polynomial Algebras and Associated Lie Algebras}

1.1. Let $A$ be an associative algebra over a field $\mathbb{F}$ and let $\sigma$ be an automorphism of $A$. Define the twisted Laurent polynomial algebra $A_{\sigma}\left[z, z^{-1}\right]$ over $A$ in the indeterminate $z$ to be the vector space $\mathbb{F}\left[z, z^{-1}\right] \otimes_{\mathbb{F}} A$ over $\mathbb{F}$ of finite sums of the form $\sum_{j \in \mathbb{Z}} z^{j} \otimes a_{j}$, $a_{j} \in A$, with multiplication defined by the rule

$$
\left(z^{k} \otimes a\right)\left(z^{m} \otimes b\right)=z^{k+m} \otimes \sigma^{m}(a) b, \quad a, b \in A, k, m \in \mathbb{Z} .
$$

Further on we shall often write $z^{m} a$ in place of $z^{m} \otimes a$.

Remarks. (a) Replacing $z$ by $z a^{-1}$, where $a$ is an invertible element of $A$, corresponds to replacing $\sigma$ by $(A d a) \sigma$, where $A d a$ stands for the inner automorphism:

$$
(A d a) b=a b a^{-1}, \quad b \in A .
$$

(b) Applying an automorphism $\alpha$ to $A$ replaces $\sigma$ by $\alpha^{-1} \sigma \alpha$.

Thus, we obtain the following proposition.

Proposition. Twisted Laurent polynomial algebras over an associative algebra $A$ are parameterized by the conjugacy classes of the group Aut $A / A d A$.

Two automorphisms of $A$ whose images lie in the same conjugacy class of Aut $A / A d A$ are called equivalent.

1.2. The algebra $A_{\sigma}\left[z, z^{-1}\right]$ has a canonical $\mathbb{Z}$-gradation, called the principal gradation:

$$
A_{\sigma}\left[z, z^{-1}\right]=\bigoplus_{j \in \mathbb{Z}}\left(z^{j} A\right) .
$$

Let $\mathscr{P}=\bigoplus_{j \in \mathbb{Z}} \mathscr{P}_{j}$ be a parabolic subalgebra of $A_{\sigma}\left[z, z^{-1}\right]$. It is clear that $\mathscr{P}_{-1}=z^{-1} I$, where $I$ is a (two-sided) ideal of the algebra $A$. Hence $\mathscr{P}$ contains the following minimal parabolic subalgebra $\mathscr{P}(I)$ associated to $I$ :

$$
\mathscr{P}(I)=\left(\bigoplus_{\jmath>0}\left(z^{-1} I\right)^{j}\right) \bigoplus\left(\bigoplus_{j \geq 0}\left(z^{j} A\right)\right) .
$$

Remark. Given two ideals $I$ and $J$ of $A$, we have the following graded subalgebra of $A_{\sigma}\left[z, z^{-1}\right]$ :

$$
\mathscr{P}(I, J)=\left(\bigoplus_{j>0}\left(z^{-1} I\right)^{j}\right) \oplus A \oplus\left(\bigoplus_{j>0}(z J)^{j}\right) .
$$

1.3. We denote $\widetilde{A}_{\sigma}$ the algebra $A_{\sigma}\left[z, z^{-1}\right]$ viewed as a Lie algebra with respect to the usual bracket:

$$
[f, g]^{\prime}=f g-g f .
$$

Fix a trace on the algebra $A$, i.e., a linear map $\operatorname{tr}: A \rightarrow V$, where $V$ is a vector space over $\mathbb{F}$, such that $\operatorname{tr}(a b)=\operatorname{tr}(b a)$. Then we may construct a remarkable central extension $\widehat{A}_{\sigma, \text { tr }}$ of $\widetilde{A}_{\sigma}$ by a central subalgebra $V$ :

$$
0 \rightarrow V \rightarrow \widehat{A}_{\sigma, \mathrm{tr}} \rightarrow A_{\sigma} \rightarrow 0
$$


as follows. It is straightforward to check that the formula

$$
\begin{aligned}
& \Psi_{\sigma, \mathrm{tr}}\left(z^{r} a, z^{s} b\right)=-\Psi_{\sigma, \mathrm{tr}}\left(z^{s} b, z^{r} a\right) \\
& \quad=\left\{\begin{array}{cl}
\operatorname{tr}\left(\left(1+\sigma+\cdots+\sigma^{r-1}\right)\left(\sigma^{-r}(f) g\right)\right) & \text { if } r=-s>0, \\
0 & \text { if } r+s \neq 0 \text { or } r=s=0
\end{array}\right.
\end{aligned}
$$

defines a 2-cocycle on $\widetilde{A}_{\sigma}$ with values in $V$. Then $\widehat{A}_{\sigma, \mathrm{tr}}=\widetilde{A}+V$ with $V$ central and the bracket of two elements $f, g \in \widetilde{A} \subset \widehat{A}_{\sigma, \text { tr }}$ is given by the usual formula:

$$
[f, g]=[f, g]^{\prime}+\Psi_{\sigma, \mathrm{tr}}(f, g) .
$$

Remarks. (a) Replacing $z$ by $z a^{-1}$ corresponds to replacing $\Psi_{\sigma, \mathrm{tr}}$ by $\Psi_{(A d a) \sigma, \mathrm{tr}}$.

(b) Applying an autormorphism $\alpha$ to $A$ replaces $\Psi_{\sigma \text {,tr }}$ by $\Psi_{\alpha^{-1} \sigma \alpha \text {,tr } \circ \alpha}$.

(c) Since $\Psi_{\sigma, \operatorname{tr}}\left(z^{r}, z^{s}\right)=\operatorname{tr}(1) r \delta_{r,-s}$, the cocycle $\Psi_{\sigma, \operatorname{tr}}$ is nontrivial if $\operatorname{tr}(1) \neq 0$.

(d) Suppose that the map $\sigma-1: A \rightarrow A$ is surjective and that $\operatorname{tr}$ vanishes on its kernel. Then we have an isomorphism $\sigma-1: A / \operatorname{Ker}(\sigma-1) \stackrel{\sim}{\rightarrow} A$, and $\varphi: \operatorname{tr} \circ(1-\sigma)^{-1}: A \rightarrow V$ is a well-defined map. We have:

$$
\Psi_{\sigma, \mathrm{tr}}\left(z^{r} f, z^{-r} g\right)=\varphi\left(\left[z^{r} f, z^{-r} g\right]\right),
$$

hence in this case the cocycle $\Psi_{\sigma, \text { tr }}$ is trivial.

(e) Suppose that $\operatorname{tr}(\sigma(a))=\operatorname{tr} a, a \in A$. Then $\operatorname{tr}$ extends to a trace of the algebra $A_{\sigma}\left[z, z^{-1}\right]$ by letting $\operatorname{tr}\left(z^{k} a\right)=\delta_{k, 0} \operatorname{tr} a$.

Example. Let $A=\mathrm{Mat}_{n} \mathbb{F}$; then any automorphism of $A$ is equivalent to $\sigma=1$ (by Remark 1(a)). Take the usual trace tr $: A \rightarrow \mathbb{F}$, then $\widehat{A}_{\sigma, \text { tr }}$ is isomorphic to the usual affine algebra $g l_{n}(\mathbb{F})^{\wedge}$.

We have the corresponding to (1.2.1) $\mathbb{Z}$-gradation:

$$
\widehat{A}_{\sigma, \mathrm{tr}}=\bigoplus_{j} \widehat{A}_{j}, \quad \text { where } \widehat{A}_{j}=z^{j} A \text { if } j \neq 0, \text { and } \widehat{A}_{0}=A+V \text {. }
$$

For each (two-sided) non-zero ideal $I$ of $A$ we have the associated parabolic subalgebra of $\widehat{A}_{\sigma, \mathrm{tr}}$,

$$
\mathfrak{p}(I)=\bigoplus_{j>0}\left(z^{-1} I\right)^{j} \bigoplus(A \bigoplus V) \bigoplus\left(\bigoplus_{j>0} z^{\jmath} A\right) .
$$

1.4. We turn now to the main examples of the Lie algebras $\widetilde{A}_{\sigma}$ and $\widehat{A}_{\sigma}$, those associated to the polynomial algebra $A=\mathbb{C}[w]$ in the indeterminate $w$. We show that the Lie algebras $\widetilde{A}_{\sigma}$ are isomorphic to the Lie algebras of all regular differential (resp. difference) operators on the punctured complex plane $\mathbb{C}^{\times}=\mathbb{C} \backslash\{0\}$, and that the Lie algebras $\widehat{A}_{\sigma, \text { tr }}$ are their well known central extensions.

For $q \in \mathbb{C}^{\times}$define the following operator on $\mathbb{C}\left[z, z^{-1}\right]$ :

$$
D_{q} f(z)= \begin{cases}\frac{f(q z)-f(z)}{q-1} & \text { if } q \neq 1 \\ z \partial_{z} f(z) & \text { if } q=1\end{cases}
$$

Denote by $\mathscr{D}_{q}^{a}$ the associative algebra of all operators on $\mathbb{C}\left[z, z^{-1}\right]$ of the form

$$
E=e_{k}(z) D_{q}^{k}+e_{k-1}(z) D_{q}^{k-1}+\cdots+e_{0}(z), \quad \text { where } e_{i}(z) \in \mathbb{C}\left[z, z^{-1}\right]
$$

(the superscript $a$ stand for "associative") and let $\mathscr{D}_{q}$ denote the corresponding Lie algebra. 
Now, any automorphism of $\mathbb{C}[w]$ is equivalent to $\sigma_{q}, q \in \mathbb{C}^{\times}$, defined by

$$
\sigma_{q}(w)=q w+1
$$

Note that

$$
\sigma_{q}^{n}(w)=q^{n} w+[n]
$$

where, as usual, for $n \in \mathbb{Z}$ :

$$
[n]=\frac{q^{n}-1}{q-1} \quad \text { if } q \neq 1 \text { and }=n \text { if } q=1 .
$$

Proposition. (a) The linear map $\mathbb{C}[w]_{\sigma_{q}}\left[z, z^{-1}\right] \rightarrow \mathscr{D}_{q}^{a}$ defined by $z^{k} f(w) \mapsto$ $z^{k} f\left(D_{q}\right)$ is an isomorphism of associative algebras.

(b) Let $\operatorname{tr}: \mathbb{C}[w] \rightarrow \mathbb{C}$ be the evaluation map at $w=0$. Then the 2-cocycle $\Psi_{\sigma, \mathrm{tr}}$ on the Lie algebra $\mathbb{C}[w]_{\sigma_{q}}\left[z, z^{-1}\right]$ induces, via the above isomorphism, the following 2-cocycle on the Lie algebra $\mathscr{D}_{q}$ :

$$
\Psi\left(z^{m} f\left(D_{q}\right), z^{n} g\left(D_{q}\right)\right)=\left\{\begin{array}{cl}
\sum_{-m \leq j \leq-1} f([j]) g([j+m]) & \text { if } m=-n \geq 0 \\
0 & \text { if } m+n \neq 0
\end{array}\right.
$$

Proof. This is straightforward using (1.4.1).

We shall denote by

$$
\widehat{\mathscr{D}_{q}}=\mathscr{D}_{q}+\mathbb{C} C
$$

the central extension of $\mathscr{D}_{q}$ corresponding to the cocycle (1.4.2) so that the bracket of two elements from the subspace $\mathscr{D}_{q}$ is given by

$$
\left[E_{1}, E_{2}\right]=E_{1} E_{2}-E_{2} E_{1}+\Psi\left(E_{1}, E_{2}\right) C \text {. }
$$

1.5. Let $\mathscr{V}^{a}=\mathscr{D}_{1}^{a}, \mathscr{D}=\mathscr{D}_{1}, \widehat{\mathscr{D}}=\widehat{\mathscr{D}}_{1, \mathrm{tr}}, D=D_{1}\left(=z \partial_{z}\right)$. As we have seen $\mathscr{D}^{a}$ is the associative algebra of all regular differential operators on the punctured complex plane $\mathbb{C}^{\times}$, i.e., operators of the form

$$
E=e_{k}(z) \partial_{z}^{k}+e_{k-1}(z) \partial_{z}^{k-1}+\cdots+e_{0}(z), \quad \text { where } e_{i}(z) \in \mathbb{C}\left[z, z^{-1}\right] .
$$

It is not difficult to see that the cocycle $\Psi$ given by (1.4.2) is given by the following formula:

$$
\Psi\left(f \partial_{z}^{m}, g \partial_{z}^{n}\right)=\frac{m ! n !}{(m+n+1) !} \operatorname{Res}_{z=0} d z f^{(n+1)}(z) g^{(m)}(z)
$$

where as usual $f^{(n)}$ stands for $\partial_{z}^{n} f$. This cocycle appeared (probably for the first time) in [KP]. It has been shown independently by several authors ([Li] and [F] among them) that $\widehat{\mathscr{D}}$ is the unique, up to isomorphism, non-trivial central extension of the Lie algebra $\mathscr{D}$ by a one-dimensional algebra.

It is, however, more convenient to write the differential operators as linear combinations of elements of the form $z^{k} f(D)$, where $f$ is a polynomial in $D$, since it is easier to compute their product [cf. (1.1.1)]:

$$
\left(z^{m} f(D)\right)\left(z^{k} g(D)\right)=z^{m+k} f(D+k) g(D) \text {. }
$$


The bracket in $\widehat{\mathscr{D}}$ is then given by

$$
\begin{aligned}
{\left[z^{r} f(D), z^{s} q(D)\right]=} & z^{r+s}(F(D+s) g(D)-f(D) g(D+r) \\
& \left.+\Psi\left(z^{r} f(D), z^{s} g(D)\right)\right) C
\end{aligned}
$$

where

$\Psi\left(z^{r} f(D), z^{s} g(D)\right)=\left\{\begin{array}{cl}\sum_{-r \leq j \leq-1} f(j) g(j+r) & \text { if } r=-s>0 \\ 0 & \text { if } r+s \neq 0 \text { or } r=s=0 .\end{array}\right.$

1.6. Consider now the associative algebra $\mathscr{D}_{q}^{a}$, the corresponding Lie algebra $\mathscr{D}_{q}$ and its central extension $\widehat{\mathscr{D}}_{q}$ in the case $q \neq 1$. Introduce the following basis of $\mathscr{D}_{q}^{a}$ :

$$
T_{m, n}=q^{\frac{1}{2}(m+1) n} z^{m}\left((q-1) D_{q}+1\right)^{n}, \quad m \in \mathbb{Z}, \quad n \in \mathbb{Z}_{+} .
$$

Then we have

$$
T_{m, n} T_{m^{\prime}, n^{\prime}}=q^{\frac{1}{2}\left(m^{\prime} n-m n^{\prime}\right)} T_{m+m^{\prime}, n+n^{\prime}}
$$

The cocycle (1.4.2) on the Lie algebra $\mathscr{D}_{q}$ becomes:

$$
\Psi\left(T_{m, n} T_{m^{\prime}, n^{\prime}}\right)=\delta_{m,-m^{\prime}} \frac{\sinh \left(\hbar m\left(n+n^{\prime}\right)\right)}{\sinh \left(\hbar\left(n+n^{\prime}\right)\right)},
$$

where we let $q=e^{2 \hbar}$. Consequently, the commutation relations of the Lie algebra $\widehat{\mathscr{D}}_{q}$ become:

$$
\begin{aligned}
{\left[T_{m, n}, T_{m^{\prime}, n^{\prime}}\right]=} & 2 \sinh \left(\hbar\left(m^{\prime} n-m n^{\prime}\right)\right) T_{m+m^{\prime}, n+n^{\prime}} \\
& +\delta_{m,-m^{\prime}} \frac{\sinh \left(\hbar m\left(n+n^{\prime}\right)\right)}{\sinh \left(\hbar\left(n+n^{\prime}\right)\right)} C
\end{aligned}
$$

Remarks. (a) Commutation relations (1.6.3) correspond to the automorphism $\sigma_{q}^{\prime}$ of $\mathbb{C}[w]$ given by $\sigma_{q}^{\prime}(w)=q w$ (which is equivalent to $\sigma_{q}$ ), and to the trace being an evaluation map at $w=1$. The evaluation map at $w=0$ gives the cocycle $\Psi_{0}\left(T_{m, n}, T_{m^{\prime}, n^{\prime}}\right)=m \delta_{m,-m^{\prime}} \delta_{n,-n^{\prime}}$, which is equivalent to $\Psi$ due to the argument of Remark 1.3(d).

(b) If we take $A=\mathbb{C}\left[w, w^{-1}\right], \sigma(x)=q x$, where $q=e^{2 \hbar} \neq 1$, and $\operatorname{tr}\left(\sum a_{i} w^{i}\right)=a_{0}$, then in the basis $T_{m, n}=q^{\frac{1}{2} m n} z^{m} w^{m}(m, n \in \mathbb{Z})$ we obtain the commutation relations of the trigonometric Sin-Lie algebra:

$$
\left[T_{m, n}, T_{m^{\prime}, n^{\prime}}\right]=2 \sinh \left(\hbar\left(m^{\prime} n-m n^{\prime}\right)\right) T_{m+m^{\prime}, n+n^{\prime}}+m \delta_{m,-m^{\prime}} \delta_{n,-n^{\prime}} C
$$




\section{Lie Algebras $\widehat{\mathscr{D}}$ and $\widehat{\mathscr{D}^{\mathscr{C}}}$.}

2.1. Let as before $D=z \partial_{z}$ and let

$$
L_{k}^{n}=z^{k} D^{n} \in \mathscr{D} \subset \widehat{\mathscr{D}} \quad\left(k \in \mathbb{Z}, n \in \mathbb{Z}_{+}\right) .
$$

Define the order and the weight by

$$
\text { ord } L_{R}^{n}=n, \quad \text { wt } L_{k}^{n}=k, \quad \text { ord } C=\text { wt } C=0 .
$$

It is clear from (1.5.4) and (1.6.1) that the order defines a filtration of $\widehat{\mathscr{D}}$ :

$$
\widehat{\mathscr{D}}^{0} \subset \widehat{\mathscr{D}^{1}} \subset \widehat{\mathscr{D}}^{2} \subset \cdots,
$$

and the weight defines the principal $\mathbb{Z}$-gradation of $\widehat{\mathscr{D}}$ :

$$
\widehat{\mathscr{D}}=\bigoplus_{j \in \mathbb{Z}} \widehat{\mathscr{D}_{j}} \text {. }
$$

Note that we have:

$$
\begin{aligned}
& \Psi\left(L_{r}^{0}, L_{s}^{0}\right)=\delta_{r,-s} r \\
& \Psi\left(L_{r}^{1}, L_{s}^{1}\right)=-\delta_{r,-s} \frac{r^{3}-r}{6}, \\
& \Psi\left(L_{r}^{0}, L_{s}^{1}\right)=\Psi\left(L_{r}^{1}, L_{s}^{0}\right)=\delta_{r,-s} \frac{r(r-1)}{2} \quad \text { if } r \geq 0 .
\end{aligned}
$$

It follows that $\widehat{\mathscr{D}}^{0}$ is isomorphic to the oscillator Lie algebra:

$$
\left[L_{r}^{0}, L_{s}^{0}\right]=\delta_{r,-s} r C .
$$

Furthermore, $\widehat{\mathscr{D}}^{1}$ contains a 1-parameter family of Virasoro algebras $\operatorname{Vir}(\beta), \beta \in \mathbb{C}$, ("complementary" to $\widehat{\mathscr{D}^{0}}$ ) defined by

$$
L_{k}(\beta)=-\left(L_{k}^{1}+\beta(k+1) L_{k}^{0}\right),
$$

so that

$$
\left[L_{r}(\beta), L_{s}(\beta)\right]=(r-s) L_{r+s}(\beta)+\delta_{r,-s} \frac{r^{3}-r}{12} C_{\operatorname{Vir}(\beta)}
$$

where

$$
C_{\operatorname{Vir}(\beta)}=\left(12 \beta^{2}-2\right) C \text {. }
$$

Remark. $z^{n+s} \partial_{z}^{n}=z^{s} D(D-1) \cdots(D-n+1)$.

2.2. Let $\odot$ be the algebra of all holomorphic functions on $\mathbb{C}$ with topology of uniform convergence on compact sets. We define a completion $\mathscr{D}^{a \mathscr{O}}$ of the (associative) algebra of differential operators on $\mathbb{C}^{\times}$by considering differential operators of infinite order of the form $z^{k} f(D)$, where $f \in \mathscr{Q}$. The usual product of differential operators extends to $\mathscr{D}^{a \mathscr{C}}$ :

$$
\left(z^{r} f(D)\right)\left(z^{s} g(D)\right)=z^{r+s} f(D+s) g(D),
$$

where by $f(D+s)$ we mean the power series expansion in $D$. The principal gradation extends as well: $\mathscr{D} a \mathscr{C}=\bigoplus_{j \in \mathbb{Z}} \mathscr{D}_{k}^{a \mathscr{C}}$, where $\mathscr{D}_{k}^{a \mathscr{C}}=\left\{z^{k} f(D) \mid f(w) \in \mathscr{O}\right\}$. Identifying 
$\mathscr{D}_{k}^{a}$ with $\mathscr{G}$ and $\mathscr{D}^{a}$ with the direct sum of $\mathscr{D}_{k}^{a}$ as topological vector spaces, we make $\mathscr{D}^{a}$ a topological associative algebra. It is a completion of the subalgebra $\mathscr{D}^{a}$.

We denote by $\mathscr{D}^{\mathscr{C}}$ the corresponding (topological) Lie algebra. Then the cocycle $\Psi$ extends by continuity from $\mathscr{D}$ to a 2 -cocycle on $\mathscr{D}^{\mathscr{G}}$ by formula (1.5.5). We let

$$
\widehat{\mathscr{D}}^{\mathscr{C}}=\mathscr{D}^{\mathscr{O}} \oplus \mathbb{C} C
$$

be the corresponding central extension. Note that for elements $z^{r} e^{\lambda D}$ $(r \in \mathbb{Z}, \lambda \in \mathbb{C})$ the commutator in $\widehat{\mathscr{D}}^{\bigcirc}$ is especially simple:

$$
\left[z^{r} e^{\lambda D}, z^{s} e^{\mu D}\right]=\left(e^{\lambda s}-e^{\mu r}\right) z^{r+s} e^{(\lambda+\mu) D}+\delta_{r,-s} \frac{e^{-\lambda r}-e^{-\mu s}}{1-e^{\lambda+\mu}} C .
$$

Remarks. (a) One may consider $z^{k} e^{\lambda D}$ as a generating series for the $L_{k}^{n}$ and derive (1.5.4) and (1.5.5) by taking derivatives of (2.2.2).

(b) Of course, $\mathscr{D}^{\mathscr{C}}$ is isomorphic to $\widetilde{\mathscr{Q}}_{\sigma_{1}}$, and $\widehat{\mathscr{D}}^{\mathscr{C}}$ to $\widehat{\mathscr{Q}}_{\sigma_{1}, \mathrm{tr}}$.

(c) Consider the following traces on $\mathscr{O}$ :

$$
\begin{aligned}
\operatorname{tr}_{a, b} f(w) & =f(a)-f(b), & & \text { where } a, b, \in \mathbb{C}, \\
\operatorname{tr}_{s}^{[m]} f(w) & =f^{(m)}(s), & & \text { where } s \in \mathbb{C}, m \in \mathbb{N} .
\end{aligned}
$$

Here and further $f^{(m)}$ stands for the $m^{\text {th }}$ derivative of $f(w)$. We denote the corresponding cocycles by $\Psi_{a, b}:=\Psi_{\sigma_{1}, \mathrm{tr}_{a, b}}$ and $\Psi_{s}^{(m)}:=\Psi_{\sigma_{1}, \mathrm{tr}_{s}^{[m]}}$. On $\mathscr{D}^{\mathscr{C}}$ these cocycles are nontrivial (in continuous cohomology). But, due to Remark 1.3(d) when restricted to $\mathscr{D}$ they become trivial. Since

$$
\left(\sigma_{1}-1\right) \frac{e^{x w}-1}{e^{x}-1}=e^{x w}
$$

using Remark 1.3(d), we obtain the following explicit formulas for these trivial cocycles on $\mathscr{D}$ :

$$
\begin{aligned}
\Psi_{a, b}\left(z^{k} f(D), z^{r} g(D)\right) & =\delta_{k,-r} \Lambda_{a, b}\left(\left[z^{k} f(D), z^{-k} g(D)\right]\right) \\
\Psi_{s}^{[m]}\left(z^{k} f(D), z^{r} g(D)\right) & =\delta_{k,-r} \Lambda_{s}^{[m]}\left(\left[z^{k} f(D), z^{-k} g(D)\right]\right)
\end{aligned}
$$

where $\Lambda_{a, b}$ and $\Lambda_{s}^{[m]}$ are the linear functions on $\mathbb{C}[w]$ defined by the following generating series in $x$ :

$$
\Lambda_{a, b}\left(e^{x w}\right)=\frac{e^{a x}-e^{b x}}{e^{x}-1}, \quad \Lambda_{x}^{(m)}\left(e^{x w}\right)=\frac{x^{m} e^{s x}}{e^{x}-1} .
$$

2.3. The following theorem describes closed ideals of $\mathscr{D}^{\mathscr{C}}$ (resp. $\widehat{\mathscr{D}}^{\mathscr{C}}$ ).

Theorem. (a) The center $Z$ of $\mathscr{D}^{\mathscr{C}}$ consists of elements of the form $f(D)$, where $f(w) \in \odot$ is a 1-periodic function (i.e., $f(w+1)=f(w))$. The center of $\widehat{\mathscr{D}}^{0}$ is $\widehat{Z}=Z \oplus \mathbb{C} C$.

(b) Let I be an ideal of $@$ which is invariant under the translation $w \mapsto w+1$, and let $I^{\prime}=\{f(D)-f(D+1) \mid f(w) \in I\}\left(\right.$ resp. $\left.\widehat{I}^{\prime}=\{f(D)-f(D+1)+f(0) C \mid f(w) \in I\}\right)$. 
Let $Y$ be a subspace of $Z$ (resp. $\widehat{Z}$ ). Let $I^{(k)}=z^{k} I \subset \mathscr{D}^{\circ}$ for $k \neq 0$ and let $I^{(0)}=I^{\prime}+Y\left(\right.$ resp. $\left.=\widehat{I}^{\prime}+Y\right)$. Then

$$
J(I, Y)=\bigoplus_{k \in \mathbb{Z}} I^{(k)}
$$

is a closed ideal of $\mathscr{D}^{\mathscr{C}}$ (resp. $\left.\widehat{\mathscr{D}^{\mathscr{C}}}\right)$.

(c) Every closed ideal of $\mathscr{D}^{\mathscr{C}}$ (resp. of $\widehat{\mathscr{D}}^{\mathscr{C}}$ ) is one of the $J(I, Y)$.

Proof. The statements (a) and (b) are clear. We shall prove (c) for $\mathscr{D}^{\mathcal{C}}$, the proof for $\widehat{\mathscr{D}}^{\mathcal{O}}$ being the same. Let $J$ be a closed ideal of the Lie algebra $\mathscr{D}^{\circ}$. Since $J$ is ad $D$-stable, it follows that $J$ is graded ideal:

$$
J=\bigoplus_{k \in \mathbb{Z}} z^{k} I_{k}
$$

where $I_{k}$ is a closed subspace of $\mathscr{O}$. We have

$$
\left[D^{2}, z^{k} f(D)\right]=2 k z^{k} D f(D)+k^{2} z^{k} f(D) .
$$

It follows that $w I_{k} \subset I_{k}$ if $k \neq 0$, i.e., that $I_{k}$ for $k \neq 0$ is an ideal of $\mathscr{Q}$. Furthermore, we let for $k \in \mathbb{Z}$ and an ideal $I$ of $\mathscr{O}$ :

$$
I[k]=\{f \in \mathscr{Q} \mid f(w+k) \in I\} .
$$

We claim that

$$
I_{k}[ \pm 1]+I_{k} \subset I_{k \pm 1} \text { if } k \neq 0 \text { and } k \pm 1 \neq 0 \text {. }
$$

Indeed, since

$$
\left[z^{ \pm 1}, z^{k} f(D)\right]=z^{k \pm 1}(f(D)-f(D \pm 1)),
$$

we see that if $f(w) \in I_{k}$ then $f(w)-f(w \pm 1) \in I_{k \pm 1}$. Since $I_{k}$ and $I_{k \pm 1}$ are ideals, $w f(w) \in I_{k}$ and $w f(w)-(w \pm 1) f(w \pm 1) \in I_{k \pm 1}$. Thus, $f(w), f(w \pm 1) \in I_{k \pm 1}$, completing the proof of $(2.3 .1)_{ \pm}$. We conclude, in particular, that

$$
I_{1}=I_{2}=\cdots \quad \text { and } \quad I_{-1}=I_{-2}=\cdots .
$$

Next, we prove that

$$
I_{k}=I_{k}[n] \text { for all } n \in \mathbb{Z}, \text { provided that } k \neq 0 .
$$

Indeed, due to (2.3.2) we may assume that $|k| \geq 2$, so that both numbers $k+1$ or $k-1$ are non-zero. Applying $(2.3 .1)_{+}$to $I_{k}$ and (2.3.1) $)_{-}$to $I_{k-1}$, we get

$$
I_{k}[1]+I_{k} \subset I_{k+1} \text { and } I_{k+1}[-1]+I_{k+1} \subset I_{k} \text {. }
$$

It follows that $I_{k}=\left(I_{k}[1]\right)[-1] \subset I_{k+1}[-1] \subset I_{k}$, and $I_{k}=\left(I_{k}[-1]\right)[1] \subset I_{k}[1] \subset$ $I_{k+1}=I_{k}$. Hence $I_{k}=I_{k}[ \pm 1]$ proving (2.3.3), which means that each $I_{k}$ is invariant under the translation $w \mapsto w+1$.

In order to complete the proof of the theorem, it remains to show that

$$
\begin{aligned}
& I_{1}=I_{-1}, \\
& I_{0} \supset I^{\prime}, \\
& I_{0} \subset I^{\prime}+Z .
\end{aligned}
$$


First, we prove that $I_{-1} \subset I_{1}$; the reverse inclusion is proved similarly. Let $f(w) \in I_{-1}$; we have:

$$
\left[z,\left[z, z^{-1} f(D)\right]\right]=z(f(D)-2 f(D+1)+f(D+2)) .
$$

Hence $f(w)-2 f(w+1)+f(w+2) \in I_{1}$. Considering $w f(w) \in I_{-1}$, we conclude that $f(w+2)-f(w+1) \in I_{1}$ and $f(w+1)-f(w) \in I_{1}$. Considering $w f(w)$ once more, we conclude that $f(w) \in I_{1}$, proving (2.3.4).

The inclusion (2.3.5) follows from the inclusions $\left[z, I_{-1}\right] \subset I_{0}$ and $\left[z^{-1}, I_{1}\right] \subset I_{0}$. Finally, in order to prove (2.3.6), note that the map $\varphi:=(\operatorname{ad} z)^{2}: z^{-1} I_{-1} \rightarrow z I_{1}$ is surjective (this follows from the proof of (2.3.4)). Let now $f \in I_{0}$ and let $g \in z^{-1} I_{-1}$ be a pre-image of $[z, f(D)]$ under the map $\varphi$. Then $[z, g] \in I^{\prime}$ and $[f-[z, g], z]=0$, hence $f-[z, g] \in Z$, proving (2.3.6).

We have the following corollary of the proof:

Corollary. The Lie algebra $\mathscr{D} / \mathbb{C}$ is simple.

2.4. Consider a parabolic subalgebra $\mathfrak{p}$ of $\widehat{\mathscr{D}}$ :

$$
\mathfrak{p}=\bigoplus_{j \in \mathbb{Z}} \mathfrak{p}_{j}, \quad \text { where } \mathfrak{p}_{\jmath}=\widehat{\mathscr{D}} \text { for } j \geq 0 \text { and } \mathfrak{p}_{\jmath} \neq 0 \text { for some } j<0 \text {. }
$$

For each positive integer $k$ we have: $\mathfrak{p}_{-k}=z^{-k} I_{-k}$, where $I_{-k}$ is a subspace of $A=\mathbb{C}[w]$. Since

$$
\left[f(D), z^{-k} P(D)\right]=z^{-k}(f(D-k)-f(D)) P(D),
$$

we see that $I_{-k}$ is an ideal of the polynomial algebra $A$. It is clear that $I_{-k} \neq 0$ for all $k=1,2, \ldots$. Let $b_{k}(w)$ be the monic (i.e., with the leading coefficient equal to 1) polynomial which is a generator of the ideal $I_{-k}$. Thus, to a parabolic subalgebra $\mathfrak{p}$ we have associated a sequence of monic polynomials $b_{1}=b_{1}(w), b_{2}=b_{2}(w), \ldots$. The polynomials $b_{k}, k=1,2, \ldots$ are called the characteristic polynomials of $\mathfrak{p}$.

Lemma. Let $\left\{b_{k}\right\}_{k \in \mathbb{N}}$ be the sequence of characteristic polynomials of a parabolic subalgebra $\mathfrak{p}$ of the Lie algebra $\widehat{\mathscr{D}}$. Then

(a) $b_{k}(w)$ divides $b_{k+1}(w)$ and $b_{k+1}(w+1)$ for all $k \in \mathbb{N}$.

(b) $b_{k+l}(w)$ divides $b_{k}(w-l) b_{l}(w)$ for all $k, l \in \mathbb{N}$.

Proof. Since $\left[z, z^{-k-1} b_{k+1}(D)\right]=z^{-k}\left(b_{k+1}(D)-b_{k+1}(D+1)\right)$, wee see that $b_{k}(w)$ divides $b_{k+1}(w)-b_{k+1}(w+1)$. Since $\left[z, z^{-k-1} D b_{k+1}(D)\right]=z^{-k}\left(D b_{k+1}(D)-(D+\right.$ $\left.1) b_{k+1}(D+1)\right)$, we see that $b_{k}(w)$ divides $w\left(b_{k+1}(w)-b_{k+1}(w+1)\right)+b_{k+1}(w+1)$, proving (a).

The proof of (b) is similar by computing the commutators $\left[z^{-k} b_{k}(D), z^{-l} b_{l}(D)\right]$ and $\left[z^{-k} b_{k}(D), z^{-l} D b_{l}(D)\right]$.

Given a monic polynomial $b(w)$, we let

$$
\begin{aligned}
b_{k}^{\min }(w) & =b(w) b(w-1) \ldots b(w-k+1), \\
b_{k}^{\max }(w) & =l c m\{b(w), b(w-1), \ldots, b(w-k+1)\} .
\end{aligned}
$$

It is easy to see that there exist (unique) parabolic subalgebras, which we denote by $\mathfrak{p}_{\min }(b)$ and $\mathfrak{p}_{\max }(b)$, for which the characteristic polynomials are $\left\{b_{k}^{\min }(w)\right\}$ and $\left\{b_{k}^{\max }(w)\right\}$ respectively. We clearly have

$$
\operatorname{dim} \widehat{\mathscr{D}}_{-k} / \mathfrak{p}_{\min }(b)_{-k}=k \operatorname{deg} b .
$$


Lemma 2.4 implies the following.

Proposition. Let $b$ be a monic polynomial and let $\mathfrak{p}$ be a parabolic subalgebra such that $b_{1}(w)=b$. Then

$$
\mathfrak{p}_{\text {min }}(b) \subset \mathfrak{p} \subset \mathfrak{p}_{\max }(b)
$$

In particular, if the difference of any two distinct roots of $b$ is not an integer, then

$$
\mathfrak{p}=\mathfrak{p}_{\min }(b)=\mathfrak{p}_{\max }(b) .
$$

Remark. $\mathfrak{p}_{\min }(b)=\mathfrak{p}((b))($ cf. $(1.3 .3))$.

2.5. Given a monic polynomial $b=b(w)$, consider the following subspace of $\widehat{\mathscr{D}}_{0}$ :

$$
\widehat{\mathscr{D}}_{0}^{b}=\{b(D) g(D)-b(D+1) g(D+1)+b(0) g(0) C \mid g(w) \in \mathbb{C}[w]\} .
$$

In order to study modules over $\widehat{\mathscr{D}}$ induced from its parabolic subalgebras, we need the following proposition.

Proposition. Let $\mathfrak{p}$ be a parabolic subalgebra of $\widehat{\mathscr{D}}$ and let $b=b(w)$ be its first characteristic polynomial. Then

$$
[\mathfrak{p}, \mathfrak{p}]=\left(\bigoplus_{k \neq 0} \mathfrak{p}_{-k}\right) \bigoplus \widehat{\mathscr{D}}_{0}^{b}
$$

In particular,

$$
\operatorname{dim} \mathfrak{p} /[\mathfrak{p}, \mathfrak{p}]=\operatorname{dim} \mathfrak{p}_{0} /[\mathfrak{p}, \mathfrak{p}]_{0}=\operatorname{deg} b(w) .
$$

Proof. Note that $[\mathfrak{p}, \mathfrak{p}]_{0}=\left[\mathfrak{p}_{1}, \mathfrak{p}_{-1}\right]$ and that $\left[z f(D+1), z^{-1} b(D) g(D)\right]$ $=b(D) f(D) g(D)-b(D+1) f(D+1) g(D+1)+b(0) f(0) g(0) C$. The rest is straightforward.

2.6. Let $\mathfrak{g}$ be a finite-dimensional semi simple Lie algebra over $\mathbb{C}$ and let $\mathfrak{g}=\bigoplus_{\alpha} \mathfrak{g}_{\alpha}$ be its root space decomposition with respect to a Cartan subalgebra $\mathfrak{g}_{0}$. An embedding $\mathfrak{g} \subset \mathscr{D}^{\mathscr{G}}$ is called graded if $\mathfrak{g}_{\alpha} \subset \mathscr{D}_{k(\alpha)}^{\mathscr{O}}$ for all $\alpha$.

Proposition. (a) The graded embeddings in $\mathscr{D}^{-}$of the Lie algebra $s_{2}(\mathbb{C})$ with the standard basis $E, H, F$ are parameterized by $k \in \mathbb{Z} \backslash\{0\}$ and by $k$-periodic functions $f, g \in \mathcal{Q}$ as follows:

$$
H=\frac{2}{k} D+f(D), \quad E=z^{k} x(D), \quad F=z^{-k} y(D),
$$

where

$$
x(D) y(D+k)=-\left(\frac{D}{k}\right)^{2}+\frac{D}{k}(f(D)+1)+g(D) .
$$

(b) The only graded embeddings of $\operatorname{sl}_{2}(\mathbb{C})$ in $\mathscr{D}$ are as follows $(k \in \mathbb{Z} \backslash\{0\}, \lambda, \mu, \in \mathbb{C})$ :

$$
H=\frac{2}{k} D+\lambda, \quad E=z^{k} x(D), \quad F=z^{-k} y(D),
$$

with the following four possibilities for $x(D)$ and $y(D)$ :

(i) $x(D)=\frac{1}{k} D^{2}+\frac{1}{k}(\lambda+1) D+\mu, y(D)=-\frac{1}{k}$ 
(ii) $x(D)=\frac{1}{k} D-\mu, \quad y(D)=-\frac{1}{k} D-\lambda-\mu-1$;

(iii) and (iv) are obtained from (i) and (ii) by the substitution $x^{\prime}(D)=y(D-k)$, $y^{\prime}(D)=x(D+k)$.

(c) A semi simple Lie algebra of rank $\geq 2$ has no graded embeddings in $\mathscr{D}^{\bullet}$.

Proof. Note that the equation

$$
\left[h(D), z^{k} x(D)\right]=\lambda z^{k} x(D)
$$

implies that

$$
h(D+k)-h(D)=\lambda
$$

All solutions of the latter equation are $h(0)=\frac{\lambda}{k} D+f(D)$, where $f(D+k)=f(D)$. Now (a) easily follows. (b) follows from (a). If rank $\mathfrak{g} \geq 2$, we always can find an element $h \in \mathfrak{g}_{0}$ such that $\alpha(h) / \beta(h)$ is an irrational number for two distinct roots $\alpha$ and $\beta$. Hence (a) implies (c).

Remarks. (a) Let $L_{n}$ denote the subalgebra of operators of $\mathscr{D}^{\bullet}$ leaving invariant the subspace $\sum_{k=0}^{n} \mathbb{C} z^{k}$ of $\mathbb{C}\left[z, z^{-1}\right]$, and let $I_{n}$ denote the ideal of $L_{n}$ of operators acting on this subspace trivially. By Jacobson's density theorem we have an exact sequence of associative algebras:

$$
0 \rightarrow I_{n} \rightarrow L_{n} \rightarrow \operatorname{Mat}_{n}(\mathbb{C}) \rightarrow 0
$$

Proposition 2.6(b) shows that this is a non-split exact sequence.

(b) It follows from the proof of the proposition that ad $\mathscr{D}_{0}^{\mathscr{O}}$ is not diagonalizable on $\mathscr{Q}^{\circ}$.

\section{Interplay between $\widehat{\mathscr{D}}$ and $\widehat{g l}(\infty)[m]$}

3.1. Let $R$ be an associative algebra over $\mathbb{C}$. Denote by $R^{\infty}$ a free $R$-module with a fixed basis $\left\{v_{j}\right\}_{j \in \mathbb{Z}}$. As usual, define the operators $E_{i j}$ by

$$
E_{\imath j} v_{k}=\delta_{\jmath k} v_{i} \text {. }
$$

Denote by $\widetilde{M}(\infty, R)$ the associative subalgebra of End $R^{\infty}$ consisting of all operators $\sum_{\imath, \jmath \in \mathbb{Z}} a_{\imath j} E_{\imath \jmath}$ whose matrices $\left(a_{i j}\right)_{i, j \in \mathbb{Z}}$ have a finite number of non-zero diagonals. Letting $\operatorname{deg} E_{\imath \jmath}=j-i$ defines the principal $\mathbb{Z}$-gradation:

$$
\widetilde{M}(\infty, R)=\bigoplus_{j \in \mathbb{Z}} \widetilde{M}(\infty, R)_{\jmath} .
$$

Fix $s \in \mathbb{C}$ and a nilpotent element $t \in R$. Consider the free $R$-module $R\left[z, z^{-1}\right] z^{s}$ and identify it with $R^{\infty}$ by choosing the basis $v_{j}=z^{-j+s}, j \in \mathbb{Z}$. By associating to an element $z^{k} f(D) \in \mathscr{D}^{a}$ the operator $z^{k} f(D+t)$ on $R\left[z, z^{-1}\right] z^{s}$, we obtain an embedding $\varphi_{s, t}: \mathscr{D}^{a} \hookrightarrow \widetilde{M}(\infty, R)$ of associative algebras over $\mathbb{C}$, which is compatible with the principal gradations. Explicitly:

$$
\varphi_{s, t}\left(z^{k} f(D)\right)=\sum_{\jmath \in \mathbb{Z}} f(-j+s+t) E_{j-k, \jmath} .
$$


The homomorphism $\varphi_{s, t}: \mathscr{D}^{a} \rightarrow \widetilde{M}(\infty, R)$ extends via (3.1.3) to a homomorphism

$$
\varphi_{s, t}: \mathscr{D}^{a} \rightarrow \widetilde{M}(\infty, R)
$$

3.2. Given a non-negative integer $m$, consider the algebra of truncated polynomials $R_{m}=\mathbb{C}[t] /\left(t^{m+1}\right)$, and let $\widetilde{M}(\infty)[m]=\widetilde{M}\left(\infty, R_{m}\right)$. We denote the homomorphism $\varphi_{s, t}: \mathscr{D}^{a \circlearrowleft} \rightarrow \widetilde{M}(\infty)[m]$ given by (3.1.3) by $\varphi_{s}^{[m]}$. By Taylor's formula we have:

$$
\varphi_{s}^{[m]}\left(z^{k} f(D)\right)=\sum_{i=0}^{m} \sum_{j \in \mathbb{Z}} \frac{f^{(i)}(-j+s)}{i !} t^{i} E_{j-k, \jmath} .
$$

Let

$$
I_{s}^{[m]}=\left\{f \in \mathscr{O} \mid f^{(i)}(n+s)=0 \text { for all } n \in \mathbb{Z} \text { and all } i=0, \ldots, m\right\},
$$

and let $J_{s}^{[m]}=\bigoplus_{k \in \mathbb{Z}} z^{k} I_{s}^{[m]} \in \mathscr{D}^{a \varrho}$. We clearly have:

$$
\operatorname{Ker} \varphi_{s}^{[m]}=J_{s}^{[m]} .
$$

Fix now $\vec{s}=\left(s_{1}, \ldots, s_{N}\right) \in \mathbb{C}^{N}$ such that $s_{i}-s_{j} \notin \mathbb{Z}$ if $i \neq j$, and fix $\vec{m}=\left(m_{1}, \ldots, m_{N}\right) \in \mathbb{Z}_{+}^{N}$. Let $\widetilde{M}(\infty)[\vec{m}]=\bigoplus_{i=1}^{M} \widetilde{M}(\infty)\left[m_{\imath}\right]$. Consider the homomorphism

$$
\varphi_{\vec{s}}^{[\vec{m}]}=\bigoplus_{\imath} \varphi_{s_{\imath}}^{\left[m_{i}\right]}: \mathscr{D}^{a C} \rightarrow \widetilde{M}(\infty)[\vec{m}]
$$

Proposition. We have an exact sequence of $\mathbb{Z}$ - graded associative algebras:

$$
0 \rightarrow J_{s}^{[\vec{m}]} \rightarrow \mathscr{D}^{a \bigodot} \stackrel{\varphi_{\vec{s}}^{[\vec{m}]}}{\longrightarrow} \widetilde{M}(\infty)[\vec{m}] \rightarrow 0
$$

where $J_{\vec{s}}^{[\vec{m}]}=\bigcap_{i=1}^{N} J_{s_{i}}^{\left[m_{\imath}\right]}$.

Proof. It is clear from (3.2.2) that $\operatorname{Ker} \varphi_{\vec{s}}^{[\vec{m}]}=J_{\vec{s}}^{[\vec{m}]}$. The surjectivity of $\varphi_{\vec{s}}^{[\vec{m}]}$ follows from the following well-known fact: for every discrete sequence of points in $\mathbb{C}$ and a non-negative integer $m$ there exists $f(w) \in \mathscr{Q}$ having prescribed values of its first $m$ derivatives at these points.

3.3. We denote by $\tilde{g l}(\infty)[m]$ the $\mathbb{Z}$-graded Lie algebra over $\mathbb{C}$ corresponding to the associative algebra $\widetilde{M}(\infty)[m]$ viewed as an algebra over $\mathbb{C}$. Consider the following 2-cocycle on $\widetilde{g l}(\infty)[m]$ with values in $R_{m}$ :

$$
C(A, B)=\operatorname{tr}[J, A] B
$$

where $J=\sum_{i \leq 0} E_{i i}$, and denote by $\widehat{g l}(\infty)[m]=\tilde{g l}(\infty)[m]+R_{m}$ the corresponding central extension. The $\mathbb{Z}$-gradation of this Lie algebra extends from $\tilde{g l}(\infty)[m]$ by letting $w t R_{m}=0$. 
The homomorphism $\varphi_{s}^{[m]}$ of the associative algebras defines a homomorphism of the corresponding Lie algebras, which we denote by the same letter:

$$
\varphi_{s}^{[m]}: \mathscr{D} \rightarrow \tilde{g l}(\infty)[m] \quad \text { and } \quad \varphi_{s}^{[m]}: \mathscr{D}^{\mathscr{C}} \rightarrow \tilde{g l}(\infty)[m] .
$$

Denote by $\Psi_{s}^{[m]}$ the restriction of the cocycle $C$ given by (2.5.1) to $\varphi_{s}^{[m]}\left(\mathscr{D}^{\mathscr{C}}\right)$. This gives us the following $R_{m}$-valued cocycle on $\mathscr{D}^{\bullet}$ :

$$
\Psi_{s}^{[m]}=\Psi+\Psi_{s, 0}+\sum_{j=1}^{m} \Psi_{s}^{(j)} \frac{t^{j}}{j !},
$$

where the cocycle $\Psi$ is given by (1.5.5) and the cocycles $\Psi_{s, 0}$ and $\Psi_{s}^{(j)}$ are defined in Remark 2.2(c). Using (2.2.4-6), we thus obtain the following proposition.

Proposition. The $\mathbb{C}$-linear map $\widehat{\varphi}_{s}^{[m]}: \widehat{\mathscr{D}} \rightarrow \widehat{g l}(\infty)[m]$ defined by

$$
\begin{aligned}
\left.\widehat{\varphi}_{s}^{[m]}\right|_{\widehat{\mathscr{U}}_{j}} & =\left.\varphi_{s}^{[m]}\right|_{\mathscr{Q}_{j}} \quad \text { if } j \neq 0, \\
\widehat{\varphi}_{s}^{[m]}\left(e^{x D}\right) & =\varphi_{s}^{[m]}\left(e^{x D}\right)-\frac{e^{s x}-1}{e^{x}-1}-\sum_{j=1}^{m} \frac{x^{j} e^{s x}}{e^{x}-1} t^{j} / j !, \\
\hat{\varphi}_{s}^{[m]}(C) & =1 \in R_{m}
\end{aligned}
$$

is a homomorphism of Lie algebras over $\mathbb{C}$.

3.4. Define an automorphism $\nu$ of the algebra $\widetilde{M}(\infty, \mathbb{C})$ by letting

$$
\nu\left(E_{i \jmath}\right)=E_{\imath+1, \jmath+1} \text {. }
$$

Let $\varphi_{s}=\varphi_{s, 0}: \mathscr{D}^{a} \rightarrow \widetilde{M}(\infty, \mathbb{C})$ (see Sect. 3.1). Then we have

$$
\varphi_{s+1}\left(z^{k} f(D)\right)=\nu \varphi_{s}\left(z^{k} f(D)\right) .
$$

Definition. A monodromic loop is a map $f: \mathbb{C} \rightarrow \widetilde{M}(\infty, \mathbb{C})$ such that

(i) $f$ is holomorphic on $\mathbb{C}$, i.e., $f(w)=\sum_{i j} f_{\imath \jmath}(w) E_{\imath \jmath}$, where $f_{i j} \in$ ?,

(ii) $f(w+1)=\nu f(w)$, i.e., $\quad f_{\imath \jmath}(w+1)=f_{\imath+1, \jmath+1}(w)$.

We let $\mathscr{L}_{\nu} \widetilde{M}(\infty)$ denote the associative algebra of all monodromic loops. It clearly inherits from $\widetilde{M}(\infty, \mathbb{C})$ the principal gradation.

Define a linear map $\varphi: \mathscr{Q}^{a C} \rightarrow \mathscr{L}_{\nu} \widetilde{M}(\infty)$ by letting

$$
\varphi(E)=\operatorname{loop}\left\{s \rightarrow \varphi_{s}(E)\right\} .
$$

This is a homomorphism of associative algebras. The inverse homomorphism $\varphi^{-1}$ is constructed as follows. Given $f(w)=\sum_{j} f_{j}(w) E_{j, j+k} \in \mathscr{L}_{\nu} \widetilde{M}(\infty)$ (a monodromic loop concentrated on the $k^{\text {th }}$ diagonal), we let

$$
\varphi^{-1}(f(w))=z^{k} f_{0}(D) .
$$

Thus we obtain the following result. 
Proposition. The map $\varphi$ is an isomorphism of $\mathbb{Z}$-graded associative algebras

$$
\varphi: \mathscr{D}^{a \varrho} \stackrel{\sim}{\rightarrow} \mathscr{L}_{\nu} \widetilde{M}(\infty)
$$

Remark. Monodromic loops are sections of the vector bundle on the cylinder $\mathbb{C} / \mathbb{Z}$ with fiber $\widetilde{M}(\infty, \mathbb{C})$ and transition function $\nu$ in a small neighbourhood of the line $\operatorname{Re} w=1$.

Denote by $\mathscr{L}_{\nu} \tilde{g l}(\infty)$ the Lie algebra obtained from $\mathscr{L}_{\nu} \widetilde{M}(\infty)$ by taking the usual bracket. For each $s \in \mathbb{C}$ define a 2-cocycle $C_{s}$ on this Lie algebra by

$$
C_{s}(f(w), g(w))=C(f(s), g(s)), \quad \text { where } f(w), g(w) \in \mathscr{L}_{\nu} \tilde{g l}(\infty)
$$

It is easy to see that under the isomorphism

$$
\varphi: \mathscr{D}^{\bigcirc} \stackrel{\sim}{\longrightarrow} \tilde{g l}(\infty)
$$

given by Proposition 2.4, the cocycle $C_{s}$ induces the following cocycle on $\mathscr{D}^{\circ}$ :

$$
\Psi_{s}\left(z^{k} f(D), z^{m} g(D)\right)=\left\{\begin{array}{cl}
\sum_{-k \leq j \leq-1} f(j+s) g(j+k+s) & \text { if } k=-m \geq 0 \\
0 & \text { if } k+m \neq 0
\end{array}\right.
$$

Denote by $\mathscr{L}_{\nu} \widetilde{g l}(\infty)^{\wedge}$ the central extension of $\mathscr{L}_{\nu} \tilde{g l}(\infty)$ corresponding to the cocycle $C_{0}$. Then the isomorphism $\varphi: \mathscr{D}^{\bullet} \stackrel{\sim}{\rightarrow} \mathscr{L}_{\nu} \underset{g l}{ } \widetilde{g}(\infty)$ lifts to the isomorphism $\widehat{\varphi}: \widehat{\mathscr{D} \mathscr{C}} \stackrel{\sim}{\rightarrow} \mathscr{L}_{\nu} \widetilde{g l}(\infty)^{\wedge}$.

\section{Quasifinite Highest Weight Modules over $\widehat{\mathscr{D}}$}

4.1. Let $b$ be a monic polynomial and let $\lambda \in \widehat{\mathscr{D}}_{0}^{*}$ be such that $\left.\lambda\right|_{\mathscr{\mathscr { V }}_{0}^{0}}=0$ (see Sect. 2.5). Consider the parabolic subalgebra $\mathfrak{p}$ whose first characteristic polynomial is $b$ and denote by $M(\lambda ; b)$ the generalized Verma module $M(\widehat{\mathscr{D}}, \mathfrak{p}, \lambda)$.

Definition. A Verma module $M(\lambda)$ over $\widehat{\mathscr{D}}$ is called highly degenerate if there exists a singular vector in $M(\lambda)_{-1}$.

The following proposition follows from Propositions 2.4 and 2.5 and formula (2.4.1).

Proposition. The following conditions on $\lambda \in \widehat{\mathscr{D}}_{0}^{*}$ are equivalent:

(i) $M(\lambda)$ is highly degenerate;

(ii) $L(\lambda)$ is quasifinite;

(iii) $L(\lambda)$ is a quotient of a generalized Verma module $M(\lambda ; b)$ for some monic polynomial $b$. 
Let $L(\lambda)$ be a quasifinite irreducible highest weight module over $\widehat{\mathscr{D}}$. According to Proposition $4.1,\left(z^{-1} b(D)\right) v_{\lambda}=0$ for some monic polynomial $b(w)$. Such monic polynomial of minimal degree is called the characteristic polynomial of $L(\lambda)$. Note that $L(\lambda)$ is a quotient of $M(\lambda ; b)$, where $b$ is the characteristic polynomial of $L(\lambda)$.

4.2. We shall characterize $\lambda \in \widehat{\mathscr{D}}_{0}^{*}$ by its labels $\Delta_{n}=-\lambda\left(D^{n}\right)$ and the central charge $c=\lambda(C)$. Introduce the generating series

$$
\Delta_{\lambda}(x)=\sum_{n=0}^{\infty} \frac{x^{n}}{n !} \Delta_{n} .
$$

Recall that a quasipolynomial is a linear combination of functions of the form $p(x) e^{\alpha x}$, where $p(x)$ is a polynomial and $\alpha \in \mathbb{C}$. Recall the following well-known fact.

Lemma. A formal power series is a quasipolynomial if and only if it satisfies a nontrivial linear differential equation with constant coefficients.

Theorem. $A \widehat{\mathscr{D}}$-module $L(\lambda)$ is quasifinite if and only if

$$
\Delta_{\lambda}(x)=\frac{\phi(x)}{e^{x}-1}
$$

where $\phi(x)$ is a quasipolynomial such that $\phi(0)=0$.

Proof. It follows from Propositions 4.1 and 2.5 that $L(\lambda)$ is quasifinite if and only if there exists a monic polynomial

$$
b(w)=w^{N}+f_{N-1} w^{N-1}+\cdots+f_{0}
$$

such that for all $s=0,1, \ldots$ we have:

$$
\lambda\left(D^{s} b(D)-(D+1)^{s} b(D+1)+b(0) \delta_{s, 0} c\right)=0 .
$$

This condition can be rewritten as follows:

$$
\sum_{n=0}^{N} f_{n} F_{n+s}=0 \text { for all } s=0,1, \ldots,
$$

where

$$
F_{n}=\delta_{n, 0} c+\sum_{j=0}^{n-1}\left(\begin{array}{l}
n \\
j
\end{array}\right) \Delta_{\jmath} .
$$

Introducing the generating series

$$
F(x)=\sum_{n=0}^{\infty} \frac{x^{n}}{n !} F_{n},
$$

we may rewrite (4.2.1) in the form

$$
\left(\sum_{n=0}^{N} f_{n}\left(\frac{d}{d x}\right)^{n}\right) F(x)=0 .
$$

Thus, by Lemma 4.2, $L(\lambda)$ is quasifinite if and only if $F(x)$ is a quasipolynomial. 
But (4.2.2) can be rewritten in terms of generating series as follows:

$$
c-F(x)=\left(e^{x}-1\right) \Delta_{\lambda}(x) .
$$

The theorem follows.

From the proof of the theorem we obtain the following.

Corollary. Let $L(\lambda)$ be a quasifinite irreducible highest weight module over $\widehat{\mathscr{D}}$, and let $b(w)$ be its characteristic polynomial. By Theorem 4.2, $F(x)=\left(1-e^{x}\right) \Delta_{\lambda}(x)+c$ is a quasipolynomial. Let $F^{(N)}+f_{N-1} F^{(N-1)}+\cdots+f_{0}=0$ be the minimal order linear differential equation with constant coefficients satisfied by $F(x)$. Then $b(w)=w^{N}+f_{N-1} w^{N-1}+\cdots+f_{0}$.

4.3. In this section we show that any quasifinite $\widehat{\mathscr{D}}$-module $V$ may be extended "by continuity" at lest to all the $\widehat{\mathscr{D}_{k}}$ for $k \neq 0$.

We shall need the following fact.

Lemma. The map $\varphi:$ (?) $\rightarrow$ given by

$$
\varphi\left(\sum_{n=0}^{\infty} f_{n} z^{n}\right)=\sum_{n=0}^{\infty}\left|f_{n}\right| z^{n}
$$

is continuous.

Proof. Given $f=\sum_{n=0}^{\infty} f_{n} z^{n} \in \mathscr{Q}$, where $f_{n}=\left|f_{n}\right| e^{i \theta_{n}}$ and $\theta_{n} \in \mathbb{R} \in \mathbb{C}$, we let

$$
f^{0}(z)=\sum_{n=0}^{\infty} e^{-\imath \theta_{n}} z^{n}, \quad f^{*}(z)=\sum_{n=0}^{\infty}\left|f_{n}\right| z^{n} .
$$

Let $B_{R}=\{z \in \mathbb{C}|| z \mid \leq R\}$ denote the disk of radius $R$ and let $C_{R}$ be its boundary. Note that $f^{0}(z)$ is holomorphic in each $B_{1-\varepsilon}$ for $0<\varepsilon<1$ and that $\max _{B_{1-\varepsilon}}\left|f^{0}(z)\right| \leq \frac{1}{\varepsilon}$.

We need to estimate $\left|f^{*}(z)\right|$ on each disk $B_{R}$. Take $R_{1}>R$ and note that for $|w|<R_{1}$ we have:

$$
f^{*}(w)=\frac{1}{2 \pi i} \int_{C_{R_{1}}} f(z) f^{0}\left(\frac{w}{z}\right) \frac{d z}{z} .
$$

From (4.3.1) we see that

$$
\max _{B_{R}}\left|f^{*}(w)\right| \leq \max _{B_{R_{1}}}|f(z)| \cdot \max _{\substack{w \in B_{R} \\|z|=R_{1}}}\left|f^{0}\left(\frac{w}{z}\right)\right| \leq \frac{1}{1-R / R_{1}} \max _{B_{1}}|f(z)| .
$$

Proposition. Let $V$ be a quasifinite $\widehat{\mathscr{D}}$-module. Then the action of $\widehat{\mathscr{D}}$ on $V$ naturally extends to the action of $\widehat{\mathscr{D}_{k}^{C}}$ on $V$ for any $k \neq 0$. Proof. Let $V=\bigoplus_{p} V_{p}$ be the $\mathbb{Z}$-gradation of $V, \operatorname{dim} V_{p}<\infty$ for all $p$. Consider the
space

$$
\operatorname{Hom}(V, V)=\bigoplus_{p, q} \operatorname{Hom}\left(V_{p}, V_{q}\right)
$$


with the topology of direct sum of finite dimensional spaces $\operatorname{Hom}\left(V_{p}, V_{q}\right)$. We can assume that the $V_{p}$ are normed spaces, and spaces $\operatorname{Hom}\left(V_{p}, V_{q}\right)$ have induced norms $\|,\|_{p, q}$.

We will show that map $\widehat{\mathscr{D}}_{k} \rightarrow \operatorname{Hom}(V, V)$ for $k \neq 0$ is continuous. To do this we have to estimate the norm of the operator $z^{k} D^{n}$ in the space $\operatorname{Hom}\left(V_{p}, V_{p+k}\right)$ for fixed $k$ and $p$ and for arbitrary $n$. We have:

$$
z^{k} D^{n}=\frac{1}{(2 k)^{n}}\left(\operatorname{ad} D^{2}-k^{2}\right)^{n} z^{k} .
$$

The operator $B=\operatorname{ad} D^{2}-k^{2}: \operatorname{Hom}\left(V_{p}, V_{p+k}\right) \rightarrow \operatorname{Hom}\left(V_{p}, V_{p+k}\right)$ acts between finite-dimensional normed spaces, hence we obtain from (4.3.2):

$$
\left\|z^{k} D^{n}\right\|_{p, p+k} \leq A \cdot \alpha^{n}, \quad \text { where } \quad A=\left\|z^{k}\right\|, \quad \alpha=\|B / 2 k\| .
$$

It follows that

$$
\begin{aligned}
\left\|z^{k} f(D)\right\|_{p, p+k} & =\left\|\sum_{n \geq 0} f_{n} z^{k} D^{n}\right\|_{p, p+k} \leq \sum_{n \geq 0}\left|f_{n}\right|\left\|z^{k} D^{n}\right\|_{p, p+k} \\
& \leq A \cdot \sum_{n \geq 0}\left|f_{n}\right| \alpha^{n}=A \varphi(f)(\alpha) .
\end{aligned}
$$

Thus, by Lemma 4.3, the map $\widehat{\mathscr{V}_{k}} \rightarrow \operatorname{Hom}(V, V)$ is continuous for $k \neq 0$. Hence this map may be extended to the completion: $\widehat{\mathscr{V}}_{k}^{\ell} \rightarrow \operatorname{Hom}(V, V)$ (the completion of $\mathbb{C}[w]$ in topology of uniform convergence on compact sets is $\mathscr{Q}$ ).

4.4. We return now to the $\mathbb{Z}$-graded complex Lie algebra $\mathfrak{g}^{[m]}:=\widehat{g} l(\infty)[m]=$ $\widetilde{g l}\left(\infty, R_{m}\right)+R_{m}$ introduced in Sect. 3.3. Recall that it is a central extension of the Lie algebra $\widetilde{g l}\left(\infty, R_{m}\right)$ over $\mathbb{C}$ by the $m+1$-dimensional space $R_{m}$. An element $\lambda \in\left(\mathfrak{g}_{0}^{[m]}\right)^{*}$ is usually given by its labels

$$
\lambda_{k}^{(j)}=\lambda\left(t^{j} E_{k k}\right), \quad k \in \mathbb{Z}, j=0, \ldots, m,
$$

and central charges

$$
c_{j}=\lambda\left(t^{j}\right), \quad j=0,1, \ldots, m \text {. }
$$

Let

$$
h_{k}^{(j)}=\lambda_{k}^{(j)}-\lambda_{k+1}^{(j)}+\delta_{k, 0} c_{\jmath}, \quad k \in \mathbb{Z}, \quad j=0, \ldots, m .
$$

As usual, we have the irreducible highest weight $\mathfrak{g}^{[m]}$-module $L\left(\mathfrak{g}^{[m]}, \lambda\right)$ associated to $\lambda$. The proof of the following proposition is standard:

Proposition. The $\mathfrak{g}_{[m]}$-module $L\left(\mathfrak{g}^{[m]}, \lambda\right)$ is quasifinite if and only if for each $j=$ $0,1, \ldots, m$ all but finitely many of the $h_{k}^{(j)}$ are zero.

4.5. Given $\vec{m}=\left(m_{1}, \ldots, m_{N}\right) \in \mathbb{Z}_{+}^{N}$, we let $\mathfrak{g}^{[\vec{m}]}=\widehat{g l}(\infty)[\vec{m}]=\bigoplus_{\imath=1}^{N} \mathfrak{g}^{\left[m_{\imath}\right]}$. By Proposition 3.3, we have a surjective homomorphism of Lie algebras over $\mathbb{C}$ :

$$
\widehat{\varphi}_{\vec{s}}^{[\vec{m}]}=\bigoplus_{i=1}^{N} \widehat{\varphi}_{s_{i}}^{\left[m_{\imath}\right]}: \widehat{\mathscr{D}} \rightarrow \mathfrak{g}^{[\vec{m}]}
$$


Choose a quasifinite $\lambda(i) \in\left(\mathfrak{g}_{0}^{\left[m_{i}\right]}\right)^{*}$ and let $L\left(\mathfrak{g}^{\left[m_{i}\right]}, \lambda(i)\right)$ be the corresponding irreducible $\mathfrak{g}^{\left[m_{i}\right]}$-module. Then

$$
L\left(\mathfrak{g}^{[\vec{m}]}, \vec{\lambda}\right)=\bigotimes_{\imath=1}^{N} L\left(\mathfrak{g}^{\left[m_{i}\right]}, \lambda(i)\right)
$$

is an irreducible $\mathfrak{g}^{[\vec{m}]}$-module. Using the homomorphism (4.5.1), we make $L\left(\mathfrak{g}^{[\vec{m}]}, \vec{\lambda}\right)$ a $\widehat{\mathscr{D}}$-module, which we shall denote by $L_{\vec{s}}^{[\vec{m}]}(\vec{\lambda})$.

We can prove now the following important Theorem.

Theorem. Consider the embedding $\widehat{\varphi}_{\vec{s}}^{[\vec{m}]}: \widehat{\mathscr{D}} \rightarrow \widehat{g l}(\infty)[\vec{m}]$ (recall that $s_{i}-s_{j} \notin \mathbb{Z}$ if $i \neq j$ ) and let $V$ be a quasifinite $\widehat{g l}(\infty)[\vec{m}]$-module. Then any $\widehat{\mathscr{D}}$-submodule of $V$ is a $\widehat{g l}(\infty)[\vec{m}]$-submodule as well. In particular, the $\widehat{\mathscr{D}}$-modules $L_{\vec{s}}^{[\vec{m}]}(\vec{\lambda})$ are irreducible.

Proof. Let $U$ be a (Z्Z-graded) $\widehat{\mathscr{D}}$ - submodule of $V . U$ is a quasifinite $\widehat{\mathscr{D}}$-module as well, hence, by Proposition 4.3, it can be extended to $\widehat{\mathscr{D}_{k}}$ for any $k \neq 0$. But by Proposition 3.2, the map $\varphi_{s}^{[\vec{m}]}: \mathscr{D}_{k}^{\varrho} \rightarrow \widetilde{g l}(\infty)[\vec{m}]_{k}$ is surjective for any $k \neq 0$. Thus $U$ is invariant with respect to all members of the principal gradation $\tilde{g l}(\infty)[\vec{m}]_{k}$ with $k \neq 0$. Since $\widehat{g l}(\infty)[\vec{m}]$ coincides with its derived algebra, this proves the theorem.

4.8. By Proposition 4.4 and Theorem 4.5, the $\widehat{\mathscr{D}}$-modules $L_{\vec{s}}^{[\vec{m}]}(\vec{\lambda})$ are irreducible quasifinite highest weight modules. Using formulas (3.2.1) and (3.3.4), it is easy to calculate the generating series $\Delta_{\vec{m}, \vec{s}, \vec{\lambda}}(x)=\sum_{n \geq 0} \Delta_{n} x^{n} / n$ ! of the highest weight and the central charge $c$ of the $\widehat{\mathscr{D}}$-module $L_{\vec{s}}^{[\vec{m}]}(\vec{\lambda})$. We have

$$
\begin{aligned}
\Delta_{m, s, \lambda}(x) & =-\sum_{j=0}^{m} \sum_{i \in \mathbb{Z}}\left(\lambda_{i}^{(j)} / j !\right) x^{j} e^{(s-i) x}+\frac{c_{0}\left(e^{s x}-1\right)+\sum_{j=1}^{m}\left(c_{j} / j !\right) x^{j} e^{s x}}{e^{x}-1} \\
c & =c_{0}
\end{aligned}
$$

and

$$
\Delta_{\vec{m}, \vec{s}, \vec{\lambda}}(x)=\sum_{\imath} \Delta_{m_{\imath}, s_{\imath}, \lambda(i)}(x), \quad c=\sum_{i} c_{0}(i)
$$

Introduce the polynomials (see (4.4.1)):

$$
g_{k}(x)=\sum_{j=0}^{m} h_{k}^{(j)} x^{j} / j ! \quad(k \in \mathbb{Z}) .
$$

Then (4.6.1) can be rewritten as follows:

$$
\Delta_{m, s, \lambda}(x)=\frac{\sum_{k \in \mathbb{Z}} e^{(s-k) x} g_{k}(x)-c_{0}}{e^{x}-1} .
$$


Using these formulas, it is not difficult to see that any irreducible quasifinite highest weight module $L(\widehat{\mathscr{V}}, \lambda)$ can be obtained in as above an essentially unique way. More precisely, we have the following.

Theorem. Let $L=L(\widehat{\mathscr{D}}, \lambda)$ be an irreducible quasifinite highest weight module with central charge $c$ and $\Delta_{\lambda}(x)=\phi(x) /\left(e^{x}-1\right)$, where $\phi(x)$ is a quasipolynomial such that $\phi(0)=0$ (see Theorem 4.2). We write $\phi(x)+c=\sum_{s \in \mathbb{C}} p_{s}(x) e^{s x}$, where $p_{s}(x)$ are polynomials. We decompose the set $\left\{s \in \mathbb{C} \mid p_{s}(x) \neq 0\right\}$ in a disjoint union of congruence classes $\bmod \mathbb{Z}$. Let $S=\left\{s, s+k_{1}, s+k_{2}, \ldots\right\}$ be such a congruence class, let $m=\max _{s \in S} \operatorname{deg} p_{s}$ and let $h_{k_{r}}^{(\jmath)}=\left(\frac{d}{d x}\right)^{\jmath} p_{s+k_{r}}(0)$. We associate to $S$ the $\widetilde{g l}(\infty)[m]$-module $L^{[m]}\left(\lambda_{S}\right)$ with the central charges

$$
c_{\jmath}=\sum_{k_{r}} h_{k_{r}}^{(j)}
$$

and labels

$$
\lambda_{i}^{(j)}=\sum_{k_{r} \geq \imath} \widetilde{h}_{k_{r}}^{(j)},
$$

where $\widetilde{h}_{k}^{(j)}=h_{k}^{(j)}-c_{j} \delta_{k, 0}$. Then the $\widehat{\mathscr{D}}$-module $L$ is isomorphic to the tensor product of all the modules $L_{s}^{[m]}\left(\lambda_{S}\right)$.

Proof. The tensor product $L^{\prime}$ of all the modules $L_{s}^{[m]}\left(\lambda_{S}\right)$ is irreducible due to Theorem 4.5. It remains to show that $L^{\prime}$ has the same highest weight as $L$ does. This is done by exploiting the observation (used already before) that $-\Delta(x)$ is the value of the highest weight of $L^{\prime}$ on $e^{x D}$, and using the formulas (4.6.2-5).

Remark. Changing the representative $s$ in $S$ amounts to the shift $\nu^{j}$ of $g l(\infty)[m]$. Up to these shifts the above construction of $L$ via the embedding $\widehat{\mathscr{V}} \rightarrow g l(\infty)[\vec{m}]$ is unique.

\section{Unitary Quasifinite Highest Weight Modules over $\widehat{\mathscr{D}}$}

5.1. It is easy to see that any anti-involution $\omega$ of the associative algebra $\mathscr{D}^{a}$, such that $\omega\left(\mathscr{D}_{\jmath}^{a}\right)=\mathscr{D}_{-\jmath}^{a}$ and $\omega(D)=D$, by a rescaling of $z$ can be brought to the following form:

$$
\omega\left(z^{k} f(D)\right)=\bar{f}(D) z^{-k}=z^{-k} \bar{f}(D-k),
$$

where for $f(D)=\sum_{i} f_{\imath} D^{\imath}$ we let $\bar{f}(D)=\sum_{i} \bar{f}_{i} D^{i}\left(f_{i} \in \mathbb{C}\right)$. The involution $\omega$ given by (5.1.1) extends to the whole algebra $\mathscr{D}^{a}$.

Note that

$$
\Psi(\omega(A), \omega(B))=\Psi(B, A), \quad A, B \in \mathscr{D}^{\mathscr{C}} .
$$

Hence the anti-involution $\omega$ of the Lie algebras $\mathscr{D}$ and $\mathscr{D}^{\mathscr{C}}$ lifts to an anti-involution of their central extensions $\widehat{\mathscr{D}}$ and $\widehat{\mathscr{D}}^{a}$, such that $\omega(C)=C$, which we again denote by $\omega$.

Remark. (a) The Virasoro subalgebra $\operatorname{Vir}(\beta)$ [defined by (2.1.7)] is $\omega$-stable if and only if $\beta=\frac{1}{2}$ 
(b) Under the homomorphism $\varphi_{s}=\varphi_{s, 0}: \mathscr{D} a \mathscr{C} \rightarrow \widetilde{M}(\infty, \mathbb{C})$ we have

$$
\left(\varphi_{s}\left(z^{k} f(D)\right)\right)^{*}=\varphi_{\bar{s}}\left(\omega\left(z^{i} f(D)\right)\right)
$$

Here $A^{*}$ stands for the complex conjugate transpose of the matrix $A \in \widetilde{M}(\infty, \mathbb{C})$. (c) (see e.g. [K]) For the involution $\omega$ of $\widehat{g l}(\infty, \mathbb{C})$ defined by $\omega(A)={ }^{t} \bar{A}, \omega(1)=1$, a highest weight $\widehat{g l}(\infty, \mathbb{C})$-module with highest weight $\lambda$ and central charge $c$ is unitary if and only if the numbers $h_{i}^{(0)}$ (see (4.4.1)) are non-negative integers and $c=\sum_{i} h_{i}^{(0)}$. 5.2. In this section we shall classify all unitary (irreducible) quasifinite highest weight modules over the Lie algebra $\widehat{\mathscr{D}}$ with respect to the anti-involution $\omega$.

Lemma. Let $V$ be a unitary quasifinite highest weight module over $\widehat{\mathscr{D}}$ and let $b(w)$ be its characteristic polynomial. Then $b(w)$ has only simple real roots.

Proof. Let $v_{\lambda}$ be a highest weight vector of $V$ and let $\Delta_{j}=-\lambda\left(D^{j}\right) \in \mathbb{R}$ be the labels of $\lambda$. Consider the element $S=-\frac{1}{2}\left(D^{2}-\Delta_{2}-1\right) \in \widehat{\mathscr{D}}$. It is easy to check that for any $j \in \mathbb{Z}_{+}$we have:

$$
S^{j}\left(z^{-1} v_{\lambda}\right)=\left(z^{-1} D^{j}\right) v_{\lambda}
$$

By definition of the characteristic polynomial we have:

$$
\begin{gathered}
\left(z^{-1} b(D)\right) v_{\lambda}=0, \\
\left\{\left(z^{-1} D^{\jmath}\right) v_{\lambda} \mid 0 \leq j<n\right\} \text { is a basis of } V_{-1},
\end{gathered}
$$

where $n=\operatorname{deg} b(w)$. It follows from (5.2.1) and (5.2.2) that

$$
b(S)\left(z^{-1} v_{\lambda}\right)=0
$$

and it follows from (5.2.3) that

$$
\left\{S^{j}\left(z^{-1} v_{\lambda}\right) \mid 0 \leq j<n\right\} \quad \text { is a basis of } V_{-1} \text {. }
$$

We conclude from (5.2.4) and (5.2.5) that $b(w)$ is the characteristic polynomial of the operator $S$ on $V_{-1}$. Operator $S$ is selfadjoint, hence roots of $b(w)$ are real.

Let $\mu$ be a root of $b(w)$ of multiplicity $m$, so that $b(w)=c(w)(w-\mu)^{m}, c(w) \in \mathbb{C}[w]$. Then

$$
v:=(S-\mu)^{m-1} c(S)\left(z^{-1} v\right)
$$

is a non-zero vector in $V_{-1}$, but

$$
h(v, v)=h\left(c(S)\left(z^{-1} v\right),(S-\mu)^{2 m-2} c(S)\left(z^{-1} v\right)\right)=0 \quad \text { if } m \geq 2
$$

by (5.2.4). Hence the unitarity forces $m=1$. 
Theorem. (a) A quasifinite irreducible highest weight module $L(\widehat{\mathscr{D}}, \lambda)$ is unitary if and only if

$$
\Delta_{\lambda}(x)=\sum_{i} n_{i} \frac{e^{s_{\imath} x}-1}{e^{x}-1}
$$

for some positive integers $n_{i}$ and real numbers $s_{i}$, such that

$$
c=\sum_{\imath} n_{\imath}
$$

(b) Any unitary quasifinite $\widehat{\mathscr{D}}$-module is obtained by taking tensor product of $N$ unitary irreducible quasifinite highest weight modules over $\widehat{g l}(\infty, \mathbb{C})$ and restricting to $\widehat{\mathscr{D}}$ via an embedding $\widehat{\varphi}_{s}^{[0]}$, where $\vec{s}=\left(s_{1}, \ldots, s_{N}\right)$ is a real vector and $s_{i}-s_{\jmath} \notin \mathbb{Z}$ if $i \neq j$.

Proof. By Proposition 4.6, being a quasifinite irreducible highest weight $\widehat{\mathscr{D}}$-module, $V$ is isomorphic to one of the modules $L_{\vec{s}}^{[\vec{m}]}(\vec{\lambda})$. It follows from Lemma 5.2 and Corollary 4.6 that $\vec{m}=0$. Now the claim (b) follows from Remarks 5.1(b) and (c). The claim (a) follows from (b) and (4.6.1 and 2).

Corollary. Suppose that only finitely many labels $\Delta_{n}$ of $\lambda$ are non-zero. Then the $\widehat{\mathscr{D}}$ module $L(\lambda)$ is unitary if and only if

$$
c=\Delta_{0} \in \mathbb{Z}_{+} \text {and } \Delta_{j}=0 \text { for } j>0 .
$$

Proof. By the hypothesis, $\Delta_{\lambda}(x)$ is a polynomial of degree $N$, where $N=$ $\max \left\{n \mid \Delta_{n} \neq 0\right\}$. By Corollary 4.2 , it follows that the characteristic polynomial of $L(\lambda)$ is $w^{N+1}$. Hence, by Lemma 5.2, $N=0$, i.e., $\Delta_{j}=0$ for $j>0$. The rest of (5.2.8) follows from Theorem 5.2.

Remark. The $\widehat{\mathscr{D}}$-modules of Corollary 5.2 are obtained by taking the embedding $\widehat{\varphi}_{0}^{[0]}: \widehat{\mathscr{D}} \rightarrow \widehat{g l}(\infty, \mathbb{C})$ and composing it with the irreducible highest weight $\widehat{g l}(\infty, \mathbb{C})$ module with a non-negative integral central charge and zero labels.

Example. Consider the following parabolic subalgebra of the Lie algebra $\mathfrak{g}=$ $\widehat{g l}(\infty, \mathbb{C})$ :

$$
\mathfrak{p}=\left\{\left(a_{\imath j}\right)_{\imath, j \in \mathbb{C}}+\mathbb{C} C \mid a_{i j}=0 \text { if } i>0 \geq j\right\}
$$

and let $F_{0}=E_{10}$. Given $c \in \mathbb{C}$, denote by $M_{c}$ the generalized Verma module $M\left(\mathfrak{g}, \mathfrak{p}, \lambda_{0}\right)$, where $\lambda_{0}$ is the highest weight such that $\lambda_{0}\left(E_{j \jmath}\right)=0$ for all $j$ and $\lambda_{0}(C)=c$. Then we have

$$
\begin{aligned}
& L\left(\mathfrak{g}, \lambda_{0}\right)=M_{c} \quad \text { if } c \notin \mathbb{Z}_{+}, \\
& L\left(\mathfrak{g}, \lambda_{0}\right)=M_{c} / \mathscr{U}(\mathfrak{g})\left(F_{0}^{c+1} v_{\lambda_{0}}\right) \quad \text { if } c \in \mathbb{Z}_{+} .
\end{aligned}
$$

Consider the homomorphism $\widehat{\varphi}_{0}: \widehat{\mathscr{D}} \rightarrow \mathfrak{g}$ given by:

$$
\widehat{\varphi}_{0}\left(z^{k} f(D)\right)=\sum_{j \in \mathbb{Z}} f(-j) E_{j-k, j}, \widehat{\varphi}_{0}(C)=1 .
$$

When restricted to $\widehat{\mathscr{D}}$, the module $L\left(\mathfrak{g}, \lambda_{0}\right)$ remains an irreducible quasifinite highest weight module with zero labels and central charge $c$. The singular vector $F_{0}^{c+1} v_{\lambda_{0}}$ 
of the $\mathfrak{g}$-module $M_{c}$ remains singular for the $\widehat{\mathscr{D}}$-module $M_{c}$. It is a multiple of the following vector:

$$
\left(z^{-1} \prod_{s=1}^{c}\left(D^{2}-s^{2}\right)\right)^{c+1} v_{\lambda_{0}} .
$$

\section{Quasifinite Highest Weight Modules over Quantum Pseudo-Differential Operators}

6.1. The $q$-analogue of the algebra $\mathscr{D}^{a}$ is the algebra $\mathscr{D}_{q}^{a}$ of all regular difference operators on $\mathbb{C}^{\times}$(see Sect. 1.4). However, a more important algebra is the algebra of quantum pseudo-differential operators $\mathscr{S}_{q}^{a}$ (which contains $\mathscr{D}_{q}^{a}$ as a subalgebra). This associative algebra is obtained by the construction explained in Sect. 1.1 by taking the algebra $A=\mathbb{C}\left[w, w^{-1}\right]$ and its automorphism $\sigma$ defined by $\sigma(w)=q w$, where $q \in \mathbb{C}^{\times}:$

$$
\mathscr{S}_{q}^{a}=A_{\sigma}\left[z, z^{-1}\right] .
$$

Explicitly, let $T_{q}$ denote the following operator on $\mathbb{C}\left[z, z^{-1}\right]$, where $q \in \mathbb{C}^{\times}$:

$$
T_{q} f(z)=f(q z) .
$$

Then $\mathscr{S}_{q}^{a}$ is the associative algebra of all operators on $\mathbb{C}\left[z, z^{-1}\right]$ of the form

$$
E=\sum_{k \in \mathbb{Z}} e_{k}(z) T_{q}^{k}, \quad \text { where } e_{k}(z) \in \mathbb{C}\left[z, z^{-1}\right] \text { and sum is finite. }
$$

As before, we write such an operator as a linear combination of operators of the form $z^{k} f\left(T_{q}\right)$, where $f$ is a Laurent polynomial in $T_{q}$. Then the product is given by

$$
\left(z^{m} f\left(T_{q}\right)\right)\left(z^{k} g\left(T_{q}\right)\right)=z^{m+k} f\left(q^{k} T_{q}\right) g\left(T_{q}\right) .
$$

Let $\mathscr{S}_{q}$ denote the Lie algebra obtained from $\mathscr{S}_{q}^{a}$ by taking the usual bracket. Let $\mathscr{S}_{q}^{\prime}=\left[\mathscr{S}_{q}, \mathscr{S}_{q}\right]$. We have:

$$
\mathscr{S}_{q}=\mathscr{S}_{q}^{\prime} \oplus \mathbb{C} T_{q}^{0} \quad \text { (direct sum of ideals). }
$$

Thus, representation theory of $\mathscr{S}_{q}$ reduces to that of $\mathscr{S}_{q}^{\prime}$.

Taking the trace form $\operatorname{tr}_{0}\left(\sum_{j} c_{j} w^{j}\right)=c_{0}$, we obtain by the general construction of Sect. 1.3 the following 2-cocycle on $\mathscr{S}_{q}$ :

$$
\Psi\left(z^{m} f\left(T_{q}\right), z^{k} g\left(T_{q}\right)\right)=m \delta_{m,-k} \operatorname{tr}_{0} f\left(q^{-m} w\right) q(w) .
$$

The associated central extension of $\mathscr{S}_{q}^{\prime}$ is denoted by $\widehat{\mathscr{S}}=\mathscr{S}_{q}^{\prime}+\mathbb{C} C$. As we have mentioned in Remark 1.6(b), this is a well-known Lie algebra studied by many authors. We will show that the representation theory of the Lie algebra $\widehat{\mathscr{S}}_{q}$ with $|q| \neq 1$ is quite similar to that of $\widehat{\mathscr{D}}$. Details of most of the proofs will be omitted, being similar as well. 
6.2. Let (2) denote (in this section) the algebra of all holomorphic functions in $\mathbb{C}^{\times}=\mathbb{C} \backslash\{0\}$. We define a completion $\mathscr{S}_{q}^{a}$ of the algebra $\mathscr{S}_{q}^{a}$ by considering operators of the form $z^{k} f\left(T_{q}\right)$, where $f \in \mathscr{O}$. We extend the product (6.1.1) to $\mathscr{S}_{q} a \mathscr{G}$ and denote by $\mathscr{S}_{q}^{a}$ the corresponding Lie algebra. The cocycle (6.1.2) extends to $\mathscr{S}_{q} \mathscr{C}$ and we let $\widehat{\mathscr{S}}_{q} \mathscr{C}=\mathscr{S}_{q}{ }^{\prime \prime}+\mathbb{C} C$ be the corresponding central extension.

Consider the associative algebra $R_{m}=\mathbb{C}[t] /\left(t^{m+1}\right)$ and let $s \in \mathbb{C}$. Then we have the following embedding $\varphi_{s, t}: \mathscr{S}_{q} a \rightarrow \widetilde{M}\left(\infty, R_{m}\right)$ of $\mathbb{Z}$-graded associative algebras over $\mathbb{C}$ (cf. [GL]):

$$
\varphi_{s}^{[m]}\left(z^{k} f\left(T_{q}\right)\right)=\sum_{j \in \mathbb{Z}} f\left(s q^{-\jmath+t}\right) E_{\jmath-k, \jmath} .
$$

It extends to a homomorphism $\varphi_{s}^{[m]}: \mathscr{S}_{q}^{a} \rightarrow \widetilde{M}(\infty, R)$.

Lemma. The homomorphism $\varphi_{s}^{[m]}$ is surjective provided that $|q| \neq 1$.

Let $\varphi_{s}=\varphi_{s}^{[0]}: \mathscr{S}_{q}^{a} \rightarrow \widetilde{M} ;(\infty, \mathbb{C})$. We have (cf. Sect. 3.4):

$$
\varphi_{q s}\left(z^{k} f\left(T_{q}\right)\right)=\nu \varphi_{s}\left(z^{k} f\left(T_{q}\right)\right)
$$

We define a quantum monodromic loop to be a holomorphic map $f: \mathbb{C}^{\times} \rightarrow \widetilde{M}(\infty, \mathbb{C})$ such that $f(q w)=\nu f(w)$. Denote by $\mathscr{L}_{q, \nu} \widetilde{M}(\infty)$ the associative algebra of all quantum monodromic loops. Then we have an isomorphism

$$
\varphi: \mathscr{S}_{q}^{a C} \stackrel{\sim}{\rightarrow} \mathscr{L}_{q, \nu} \widetilde{M}(\infty)
$$

defined by the same formula as (3.4.2). Note that the quantum monodromic loops are sections of the vector bundle on the tours $\mathbb{C}^{\times} /\left\{q^{n} \mid n \in \mathbb{Z}\right\}$ (with modular parameter $(\log q) /(2 \pi i))$ with fiber $\widetilde{M}(\infty, \mathbb{C})$ and transition function $\nu$ in a small neighbourhood of the circle $|w|=|q|$.

Denote by $\mathscr{L}_{q, \nu} \tilde{g l}(\infty)$ the Lie algebra obtained from $\mathscr{L}_{q, \nu} \widetilde{M}(\infty)$ by taking the usual bracket. Considering the Laurent expansion at 0 :

$$
C(f(w), g(w))=\sum_{n \in \mathbb{Z}} C_{n}(f, g) w^{n}
$$

we obtain $\mathbb{C}$-valued 2-cocycles $C_{n}$ on this Lie algebra. Denote by $\mathscr{S}_{q, \nu} \tilde{g l}(\infty)^{\wedge}$ the corresponding to $C_{0}$ central extension. Then the isomorphism $\varphi: \mathscr{S}_{q} \stackrel{\stackrel{\sim}{\rightarrow}}{\rightarrow} \mathscr{C}_{q, \nu} \widetilde{g l}(\infty)$ lifts to the isomorphism $\widehat{\varphi}: \widehat{\mathscr{P}}_{q}{ }^{\mathscr{C}} \stackrel{\sim}{\rightarrow} \mathscr{L}_{q, \nu} \widetilde{g l}(\infty)^{\wedge}$.

6.3. Let $\mathfrak{p}=\bigoplus_{j \in \mathbb{Z}} \mathfrak{p}_{j}$ be a parabolic subalgebra of the Lie algebra $\widehat{\mathscr{S}_{q}}$ (i.e., $\mathfrak{p}_{j}=\left(\widehat{\mathscr{S}}_{q}\right)_{j}$ for $j \geq 0$ and $\left(\widehat{\mathscr{S}}_{q}\right)_{\jmath} \neq 0$ for some $\left.j<0\right)$. Then for each positive integer $k$ we have $\mathfrak{p}_{-k}=z^{-k} I_{-k}$, where $I_{-k}$ is a non-zero ideal of $\mathbb{C}\left[w, w^{-1}\right]$. Let $b_{k}(w)$ be the monic polynomial with $b_{k}(0) \neq 0$ which is a generator of the ideal $I_{-k}$. The polynomials $b_{k}, k=1,2, \ldots$ are called the characteristic polynomials of $\mathfrak{p}$. 
Given a monic polynomial $b(w)$ with $b(0) \neq 0$, we let

$$
\begin{aligned}
b_{k}^{\min }(w) & =b(w) b\left(q^{-1} w\right) \cdots b\left(q^{-k+1} w\right), \\
b_{k}^{\max } & \left.=\operatorname{lcm}\left\{b(w), b\left(q^{-1} w\right), \ldots, b\left(q^{-k+1}\right) w\right)\right\}
\end{aligned}
$$

There exist unique parabolic subalgebras of $\widehat{\mathscr{S}_{q}}$, which we denote by $\mathfrak{p}_{\min }(b)$ and $\mathfrak{p}_{\max }(b)$, for which the characteristic polynomials are $\left\{b_{k}^{\min }(w)\right\}$ and $\left\{b_{k}^{\max }(w)\right\}$ respectively. We have

$$
\operatorname{dim}\left(\widehat{S}_{q}\right)_{-k} / \mathfrak{p}_{\min }(b)_{-k}=k \operatorname{deg} b(w),
$$

and an analogue of Proposition 2.4 holds verbatim.

Also, we have for a parabolic subalgebra $\mathfrak{p}$ with the first characteristic polynomial $b(w)$ :

$$
[\mathfrak{p}, \mathfrak{p}]=\left(\bigoplus_{k \neq 0} \mathfrak{p}_{k}\right) \bigoplus\left(\widehat{S}_{q}\right)_{0}^{b}
$$

where

$$
\left(\widehat{\mathscr{S}}_{q}^{b}\right)_{0}^{b}=\left\{b\left(T_{q}\right) g\left(T_{q}\right)-b\left(q T_{q}\right) g\left(g T_{q}\right)+\left(\operatorname{tr}_{0} b(w) g(w)\right) C \mid g(w) \in \mathbb{C}\left[w, w^{-1}\right]\right\} .
$$

6.4. All the results of Sect. 4.1 hold for the Lie algebra $\widehat{\mathscr{S}}_{q}$ verbatim. However, the generating series $\Delta_{\lambda}(x)$ is defined differently.

We shall characterize $\lambda \in\left(\widehat{\mathscr{S}}_{q}\right)_{0}^{*}$ by labels $\Delta_{n}=\lambda\left(T_{q}^{n}\right)(n \neq 0)$ and central charge $c=\lambda(C)$. Introduce the generating series

$$
\Delta_{\lambda}(x)=\sum_{\substack{n \in \mathbb{Z} \\ n \neq 0}} \Delta_{n} x^{-n} .
$$

Theorem. (a) An irreducible highest weight module $L\left(\widehat{S}_{q}, \lambda\right)$ is quasifinite if and only if one of the following equivalent conditions holds:

(i) There exists a non-zero polynomial $b(x)$ such that

$$
b(x)\left(\Delta_{\lambda}(x)-\Delta_{\lambda}\left(q^{-1} x\right)+c\right)=0 .
$$

(ii) There exists a quasipolynomial $P(x)$ such that

$$
\left(1-q^{n}\right) \Delta_{n}=P(n) \text { for } n \neq 0 \text { and } c=P(0) .
$$

(b) The monic polynomial of minimal degree satisfying (6.4.1) is the characteristic polynomial of a quasifinite module $L\left(\widehat{\mathscr{S}}_{q}, \lambda\right)$.

Proof. According to Sect. $6.3, L\left(\widehat{\mathscr{S}}_{q}, \lambda\right)$ is quasifinite if and only if there exists a non-zero polynomial $b(w)$ such that

$$
\lambda\left(g\left(T_{q}\right) b\left(T_{q}\right)-g\left(q T_{q}\right) b\left(q T_{q}\right)\right)+c \operatorname{tr}_{0}(g(w) b(w))=0
$$


for each $g(w) \in \mathbb{C}\left[w, w^{-1}\right]$. Taking $g(w)=w^{n}$, and letting $b(w)=\sum_{j} f_{\jmath} w^{j}$, this can be rewritten as follows:

$$
\sum_{\jmath} f_{j} \Delta_{n+j}\left(1-q^{n+j}\right)+f_{-n} c=0 \quad \text { for all } n \in \mathbb{Z} .
$$

Multiplying both sides of this equality by $x^{-n}$ and summing over $n \in \mathbb{Z}$, we obtain (6.4.1).

The equivalence of (i) and (ii), as well as (b) are clear.

6.5. Choose a branch of $\log q$. Let $\tau=(\log q) /(2 \pi i)$. Then any $s \in \mathbb{C}$ is uniquely written as $s=q^{a}, a \in \mathbb{C} / \tau^{-1} \mathbb{Z}$. The homomorphism $\varphi_{s}^{[m]}: \mathscr{S}_{q}^{\prime} \rightarrow \widetilde{g l}(\infty)[m]$ defined by (6.2.1) lifts to a homomorphism $\widehat{\mathscr{S}_{q}} \rightarrow \widehat{g l}(\infty)[m]$ of central extensions, denoted by $\widehat{\varphi}_{a}^{[m]}$, by

$$
\begin{aligned}
\left.\widehat{\varphi}_{a}^{[m]}\right|_{\left(\widehat{.}()_{j}\right.} & =\left.\varphi_{a}^{[m]}\right|_{\left(\widehat{S_{q}}\right)_{j}} \quad \text { if } j \neq 0, \\
\widehat{\varphi}_{a}^{[m]}\left(T_{q}^{n}\right) & =\sum_{r \in \mathbb{Z}} q^{(a-r)} E_{r r}+\frac{q^{a n}}{1-q^{n}} \sum_{j=0}^{m}(n \log q)^{\jmath} t^{\jmath} / j ! \quad(n \neq 0), \\
\widehat{\varphi}_{a}^{[m]}(C) & =1 \in R_{m} .
\end{aligned}
$$

We have results similar to Theorems 4.5, 4.6, and 5.2:

Theorem. Assume that $|q| \neq 1$. Consider the embedding $\widehat{\varphi}_{\vec{a}}^{[\vec{m}]}: \widehat{\mathscr{C}}_{q} \rightarrow \widehat{g l}(\infty)[\vec{m}]$, where $a_{i}-a_{j} \notin \mathbb{Z}+\tau^{-1} \mathbb{Z}$ if $i \neq j$. Denote the quasifinite $\widehat{g l}(\infty)[\vec{m}]$-module $L^{[\vec{m}]}(\vec{\lambda})$, viewed as a $\widehat{\mathscr{S}}_{q}$-module via this embedding, by $L_{\vec{a}}^{[\vec{m}]}(\vec{\lambda})$.

(a) If $V$ is a quasifinite $\widehat{g l}(\infty)[\vec{m}]$-module, then any submodule of the module $V$, viewed as a $\widehat{\mathscr{S}}_{q}$-module via the embedding $\widehat{\varphi}_{\vec{a}}^{[\vec{m}]}$, is a $\widehat{g l}(\infty)[\vec{m}]$ submodule as well. In particular, the $\widehat{\mathscr{S}}_{q}$-modules $L_{\vec{a}}^{[\vec{m}]}(\vec{\lambda})$ are irreducible.

(b) Any irreducible quasifinite highest weight module over $\widehat{\mathscr{S}}_{q}$ is isomorphic to one of the modules $L_{\vec{a}}^{[\vec{m}]}(\vec{\lambda})$.

(c) Let $q \in \mathbb{R}$, and let $\omega$ be the anti-involution of $\widehat{\mathscr{S}_{q}}$ defined by

$$
\omega\left(z^{k} f\left(T_{q}\right)\right)=z^{-k} \bar{f}\left(q^{-k} T_{q}\right) .
$$

Then a quasifinite highest weight module over $\widehat{\mathscr{S}}_{q}$ is unitary with respect to $\omega$ if and only if

$$
\Delta_{n}=\sum_{\jmath} \frac{n_{\jmath} q^{a_{\jmath} n}}{1-q^{n}} \quad \text { (finite sum) for all } n \neq 0,
$$

where the $n_{j}$ are positive integers and the $a_{j}$ are real numbers. (In particular, unitarity implies that $c=\sum_{j} n_{j} \in \mathbb{Z}_{+}$.) Any unitary quasifinite $\widehat{\mathscr{S}_{q}}$-module is obtained by taking the tensor product of $N$ unitary irreducible quasifinite highest weight modules over $\widehat{g l}(\infty, \mathbb{C})$ and restricting to $\widehat{\mathscr{S}}_{q}$ via an embedding $\widehat{\varphi}_{\vec{a}}^{[0]}, \vec{a} \in \mathbb{R}^{N}$. 
Remarks. (a) The labels and the central charge of the $\widehat{\mathscr{S}}_{q}$-module $L_{\vec{a}}^{[m]}(\lambda)$ are given by the following formulas (see (4.6.4)):

$$
\Delta_{n}=\sum_{k \in \mathbb{Z}} q^{(a-k) n} g_{k}(n \log q) /\left(1-q^{n}\right), \quad n \neq 0 ; \quad c=c_{0} .
$$

(b) A vertex operator construction of unitary quasifinite highest weight modules over $\widehat{\mathscr{S}}_{q}$ with $c=1$ is given in [GL].

\section{Matrix Case}

Let us consider the Lie algebras $M_{n} \mathscr{D}=\operatorname{Mat}_{n}(\mathscr{D})$ and $M_{n} \mathscr{S}_{q}$. They are twisted Laurent polynomial algebras with $A=\operatorname{Mat}_{n}[w], \sigma(w)=w+1$ and $A=$ $\operatorname{Mat}_{n}\left[w, w^{-1}\right], \sigma(w)=q w$ respectively.

The canonical central extension $M_{n} \widehat{\mathscr{S}_{q}}$ is defined via (1.3.1) with respect to the trace functional $\operatorname{tr}_{0}: \operatorname{Mat}_{n}\left[w, w^{-1}\right] \rightarrow \mathbb{C}$ defined by

$$
\operatorname{tr}_{0}\left(\sum_{i} m_{\imath} w^{\imath}\right)=\operatorname{tr} m_{0}
$$

Restriction of $\operatorname{tr}_{0}$ to $\operatorname{Mat}_{n}[w]$ gives rise to the canonical central extension $M_{n} \mathscr{D}^{\wedge}$. The isomorphism $\mathbb{C}^{n}\left[z, z^{-1}\right] \stackrel{\sim}{\rightarrow} \mathbb{C}\left[z, z^{-1}\right]$ defined by

$$
e_{i} z^{\jmath} \rightarrow z^{\jmath n+i}
$$

defines the isomorphism $\operatorname{Mat}_{n}(\widetilde{M}(\infty)) \stackrel{\sim}{\longrightarrow} \widetilde{M}(\infty)$. Combined with isomorphism $\widehat{\mathscr{D}}^{\mathcal{O}} \stackrel{\sim}{\rightarrow} \mathscr{L}_{\nu} \tilde{g l}(\infty)^{\wedge}$ and $\mathscr{S}_{q} \stackrel{\sim}{\sim} \underset{\sim}{\rightarrow} \mathscr{L}_{q, \nu} \widetilde{g l}(\infty)^{\wedge}$ it gives Lie algebra isomorphisms:

$$
\begin{array}{rlrl}
M_{n} \mathscr{D} \mathscr{C} \wedge & \stackrel{\sim}{\rightarrow} \mathscr{L}_{\nu} \operatorname{Mat}_{n} \tilde{g l}(\infty)^{\wedge} & \stackrel{\sim}{\rightarrow} \mathscr{L}_{\nu^{n}} \tilde{g l}(\infty)^{\wedge}, \\
M_{n} \mathscr{S}_{q} \stackrel{\sim}{\rightarrow} \mathscr{L}_{q, \nu} \operatorname{Mat}_{n} \tilde{g l}(\infty)^{\wedge} & \stackrel{\sim}{\rightarrow} \mathscr{L}_{q, \nu^{n}} \tilde{g l}(\infty)^{\wedge} .
\end{array}
$$

The representation theory of the Lie algebras $M_{n} \mathscr{D}^{\wedge}$ and $M_{n} \mathscr{S}_{q} \wedge$ is similar to that of $\widehat{\mathscr{D}}$ and $\widehat{\mathscr{S}_{q}}$. All irreducible quasifinite highest weight modules over $M_{n} \mathscr{D}^{\wedge}$ and $M_{n} \mathscr{S}_{q}^{\wedge}$ are constructed by embedding in $\widehat{g l}(\infty)[\vec{m}]$ and restricting an irreducible quasifinite highest weight module over the latter.

An anti-involution of an algebra $B$ combined with the matrix transposition defines an anti-involution of $\operatorname{Mat}_{n}(B)$. All quasifinite unitary highest weight modules over $M_{n} \mathscr{D}^{\wedge}$ and $M_{n} \mathscr{S}_{q}^{\wedge}$ are modules over $g l(\infty)[\overrightarrow{0}]^{\wedge}$. In particular, unitary modules over $M_{n} \mathscr{D}^{\wedge}$ and $M_{n} \mathscr{S}_{q}^{\wedge}$ have positive integer central charge.

\section{References}

[F] Feigin, B.L.: The Lie algebras $g l(\lambda)$ and the cohomology of the Lie algebra of differential operators. Usp. Math. Nauk 35, No. 2, 157-158 (1988)

[FL] Luk'yanov, S.L., Fateev, V.A.: Conformally invariant models of two-dimensional quantum field theory with $Z_{n}$ symmetry. Zh. Esp. Theor. Fiz. 94, No. 3, 23-37 (1988)

[FFZ] Fairlie, D., Fletcher, P., Zachos, C.: Phys. Lett. 218B, 203 (1989)

[GL] Golenishcheva-Kutuzova, M., Lebedev, D.: Vertex operator representation of some quantum tori Lie algebras. Commun. Math. Phys. 148, 403-416 (1992) 
[K] Kac, V.G.: Infinite-dimensional Lie algebras. 3-d edition, Cambridge: Cambridge University Press, 1990

[KP] Kac, V.G., Peterson, D.H.: Spin and wedge representations of infinite-dimensional Lie algebras and groups, Proc. Natl. Acad. Sci. USA 78, 3308-3312 (1981)

[KhZ] Khesin, B., Zakharevich, I.: Poisson-Lie group of pseudodifferential symbols and fractional KP-KdV hiearchies. C.R. Acad. Sci. Paris, t.316, Serie 1, 621-626 (1993)

[Li] Li, W.L.: 2-cocycles on the algebra of differential operators. J. Algebra 122, 64-80 (1989)

[PSR] Pope, C.N., Shen, X., Romans, L.J.: $W_{\infty}$ and the Racah-Wigner Algebra. Nucl. Phys. 339B, 191-122 (1990)

[R] Radul, A.O.: Lie algebras of differential operators, their central extensions and $W$-algebras. Funct. Anal. Appl. 25, No. 1, 86-91 (1991)

[RV] Radul, A.O., Vaysburd, I.: Differential operators and $W$-algebras. Phys. Lett. 274B, 317-322 (1992)

[Z] Zamolodchikov, A.B.: Infinite additional symmetries in 2-dimensional conformal quantum field theory. Teor. Mat. Phys. 65, 1205-1213 (1985)

Communicated by A. Jaffe 
